\title{
EXPERIMENTAL, THEORETICAL AND COMPUTATIONAL STUDIES OF THE PLASMA BEAT WAVE ACCELERATOR AT UCLA
}

\author{
C. Joshi, T. Katsouleas, C. E. Clayton, W. B. Mori, \\ J. M. Kindel*, and D. W. Forslund** \\ University of California, Los Angeles
}

\begin{abstract}
1. Introduction

2. Experimental Program

3. Theory Program

4. Simulations Program

5. Summary
\end{abstract}

In this paper we report on the recent progress on the Plasma Beat Wave Accelerator Scheme at UCLA as described in a series of talks by the authors at the Symposium on Advanced Accelerator Concepts in Madison. This paper is organized as follows:

\section{INTRODUCTION}

The University of California at Los Angeles has a comprehensive "Plasma Accelerators" research program with a balanced emphasis on experiments, theory and computer simulations. This program is assessing the possible role of plasmas in future high-energy accelerators. At present, we are working on two novel plasma accelerator schemes known as the Plasma Beat Wave Accelerator (PBWA) and the Plasma Wake Field Accelerator (PWFA). In addition to this, we are studying the possibility of using plasmas for focusing ultra-high energy particles.

In the PBWA, two laser beams of slightly different frequencies resonantly beat in a plasma, in such a way that their frequency and wavenumber differences correspond to the plasma wave frequency and wavenumber ${ }^{(1)}$. The amplitude modulated beat wave exerts a periodic ponderomotive force on the plasma electrons, causing them to bunch. The resulting space charge wave has a phase velocity that is equal to the group velocity of the beating waves. If the laser frequencies are much greater than the plasma frequency, the group velocity is nearly $c$. If an electron is now injected with a velocity close to this, it can be trapped and accelerated much in the same way as a surfer riding an ocean wave.

In the PWFA, a high-current but low-voltage electron bunch is used to excite the plasma wave ${ }^{(2)}$. The phase velocity of this plasma wave (like the wake of a boat) is tied to the velocity of the driving bunch, which is close to $c$. This wave then accelerates a trailing, low-density bunch to high voltage or energy. The plasma thus acts as a transformer,

In collaboration with: J. M. Dawson, F. F. Chen, P. Chen, J. J. Su, S. Wilks, K. Marsh, C. Darrow D. Umstadter, H. Figueroa and R. Williams.

* J. M. Kindel, Los Alamos National Laboratory and Mission Research Corporation, Los Alamos, NM.

** D. W. Forslund, Los Alamos National Laboratory, Los Alamos, NM. 
increasing voltage at the expense of current. The key to obtaining a high transformer ratio is to use a slowly ramped but sharply truncated (compared to $c / \omega_{p}$ ) driving beam.

The detailed description of the PWFA can be found elsewhere in these proceedings ${ }^{(3)}$. It should be emphasized that the accelerating mechanism in both the PBWA and the PWFA is identical; only the energy source which drives the relativistic plasma wave is different. A comprehensive discussion on the fraction of the energy that can be transferred from the plasma wave to the accelerating particles (the so-called beam-loading efficiency) is therefore included in this paper.

At the interaction point of a TeV class $\mathrm{e}^{-}-e^{+}$collider, the colliding beams will be tens of angstroms in diameter in order to achieve the necessary luminosity. A plasma lens may be able to provide sufficient focusing strength necessary to achieve this in a reasonable distance. The basic mechanism of a plasma lens is as follows: As a bunch of high energy electrons, which is to be focused, enters a high density plasma, it expels some or all of the plasma electrons. The space charge force within the bunch is now reduced because of the plasma ions and the bunches' own $\mathrm{i} \times \underline{\mathrm{B}}$ force can now pinch it . For plasma densities of the order $10^{16} \mathrm{~cm}^{-3}$ the focusing field strengths can be as high as $100 \mathrm{MG} / \mathrm{cm}$ on a $15 \mu \mathrm{m}$ radius bunch. In this paper we report on some preliminary simulations studies of the plasma lens.

\section{EXPERIMENTAL PROGRAM}

The main objectives of the UCLA group's experimental program on the PBWA have been as follows:

1) To study the physics of the beat wave excitation with nanosecond long, $\mathrm{CO}_{2}$ laser pulses.

2) To identify competing plasma instabilities and develop techniques to eliminate these deleterious effects.

3) Study the saturation mechanism(s) of the beat excited plasma wave.

4) To estimate the laser to plasma wave coupling efficiency.

5) To develop suitable laser and plasma sources particularly suited to the beat wave program.

6) To demonstrate, in a controlled fashion, the acceleration of externally injected test particles and confirm the promise of ultra-high accelerating gradients achievable by using plasma waves.

We will now discuss each of these objectives in some detail. In the proceedings of the Malibu Workshop ${ }^{(4)}$ we discussed in great detail our experiments that demonstrated gradients of $\approx 1 \mathrm{GeV} / \mathrm{m}$ in a $10^{17}$ plasma excited using $9.6 \mu \mathrm{m}$ and $10.6 \mu \mathrm{m}$ lines of a $\mathrm{CO}_{2}$ laser. Readers are referred to refs. $(1,4)$ for further details. The laser pulses used in these experiments were approximately $1.5 \mathrm{~ns}$ long and had an intensity of $\approx 10^{13} \mathrm{~W} / \mathrm{cm}^{2}$. With 
such intense and long laser pulses, the beat wave effect is not isolated. The laser beam can decay via the stimulated Brillouin instability (SBS) into a backward propagating e.m. wave and an ion wave. In a very underdense plasma $\omega_{0}, \omega_{1} \gg \omega_{p}$, the ion wave has a wavenumber approximately twice that of the laser beams. This ion wave effectively "ripples" the otherwise quasi-homogeneous plasma on a fine scale. In our previously reported work we had used the frequency of the ion wave to estimate the plasma temperature. Another instability which we had observed was the stimulated Raman scatter (SRS) in which the incident laser decays into a backscattered e.m. wave and a plasma wave with a wavenumber approximately the same as that of the ion wave. This plasma mode which has frequency $\approx \omega_{\mathrm{p}}$ was used to diagnose the plasma density. Although these competing instabilities are very useful for diagnosing the plasma parameters they are not desirable in the PBWA because they can act as an energy loss mechanism for the pump laser beams, produce noise in the plasma and saturate the beat excited plasma wave to an amplitude well below what might be expected in a homogeneous plasma. In our present series of experiments therefore we plan to use a much shorter laser pulse (on the order of 100-200 picoseconds) in conjunction with a plasma which has an ion temperature greater than the electron temperature to suppress the SBS instability. A high power ( $100 \mathrm{GW}$ ) $\mathrm{CO}_{2}$ laser is being developed especially for this purpose and will be described later in this section.

Saturation of the beat excited plasma wave by mode coupling. As mentioned in the previous paragraph with nanosecond long laser pulses beat wave excitation occurs in a rippled plasma. Consequently, as the dispersion relation plotted in Fig. 1 shows, there is not a unique value of $k_{p}$ for a given value of $\omega_{\text {epw. }}$. When one considers the nature of plasma

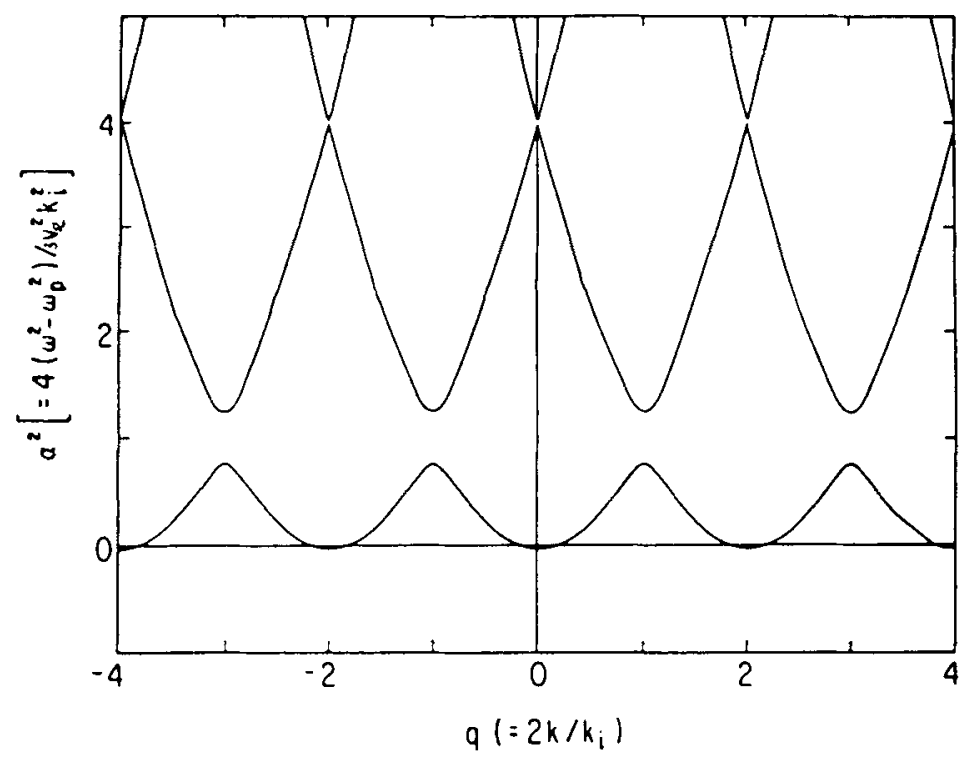

Fig. 1. Dispersion relation of an electron plasma wave in a "rippled" plasma with a ripple wavenumber $\mathrm{k}_{\mathrm{i}}$. 
waves which are resonantly excited in a rippled-density plasma, one finds that the plasma wave of frequency $\omega_{e p w}$ and wavenumber $k_{p}$ couples to a number of secondary electrostatic modes of the same frequency but wavenumbers $k_{p} \pm n k_{i}$, where $k_{i}$ is the ripple wavenumber. This electrostatic mode coupling can saturate the beat wave at an amplitude below that given by relativistic detuning.

We have investigated this mode coupling process both experimentally and theoretically. Figure 2 shows the numerical solution to the Eulerian equation for the plasma wave electric field, with and without the ripple, when driven by the ponderomotive force of the beating lasers. The beat wave driver is incident from the left and has just reached the right-hand boundary. For the homogeneous case (Fig. 2a) the plasma wave grows secularly in time and the Fourier spectrum shows a single peak at $\mathbf{k}=\mathrm{k}_{\mathrm{p}}$ as expected. In the rippled case, the higher $k$ components are excited at $k_{p} \pm n k_{i}$ and (although the peak amplitude of the waveform still exhibits secular growth with the same growth rate), the relative size of the $k_{p}$ component is reduced.

Neglecting pump risetimes we found that saturation of the beat excited plasma wave by mode coupling dominates over that due to relativistic detuning whenever

$$
\alpha_{1} \alpha_{2}<[1.6 \varepsilon / f(\mathrm{p})]^{3 / 2}
$$

where $f(p)=p+\left(1+p^{2}\right)\left(2+p^{2}\right)^{-1 / 2}$ and $p=(3 / \varepsilon)\left(k_{i} \lambda_{d}\right)^{2}$. For instance, in the case of a modest ripple size of $4 \%$ (as in our experiments), we found that saturation by mode coupling dominates unless $\alpha>0.04$ or I $\left(\mathrm{CO}_{2}\right)>4 \times 10^{13} \mathrm{~W} / \mathrm{cm}^{2}$. Here $\alpha=v_{d} / \mathrm{c}$, is the normalized oscillating velocity of the electron in the laser field. Since our experiments were carried out at an intensity of $\approx 10^{13} \mathrm{~W} / \mathrm{cm}^{2}$ we expected to see the evidence of mode coupling.

By scanning the Thomson scattering angle and thereby $\underline{\mathbf{k}}$ matching to different electrostatic modes we mapped out the complete electrostatic spectrum. This is shown in Fig. 3. Here the modes marked $n \omega_{i}, n=1,2,3$ are the fundamental and the harmonics of the ion acoustic wave from SBS, a clear indiction of the ripple being present. The mode at frequency $\omega_{p}$ and wavenumber $k_{p}$ is the beat driven plasma wave. The other modes at frequency $\omega_{p}$ and wavenumbers approximately $\pm n k_{i}$ are the signature of electrostatic mode coupling draining energy away from the beat driven plasma wave into low phase velocity modes which can subsequently be Landau damped. Beat wave saturation by mode coupling can be avoided by the elimination of the ripple (short laser pulses or $T_{i} \geq T_{e}$ plasmas). We intend to use both methods in the next series of experiments designed to demonstrate particle acceleration. First, we intend to use much shorter laser pulses, on the order of $100 \mathrm{ps}$ risetime to minimize the ion plasma periods over which the beat wave is excited. Second, we will use a $\theta$-pinch plasma source in which a homogeneous column of plasma which has $T_{i}>T_{e}$ is confined by a magnetic field. By combining these two techniques we hope to suppress the SBS instability and thereby not have a rippled plasma. 
(a)

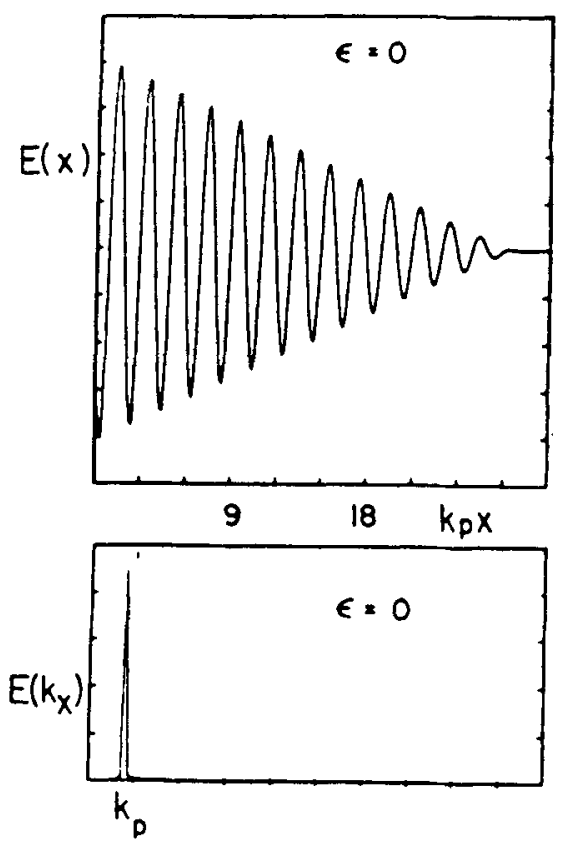

(b)

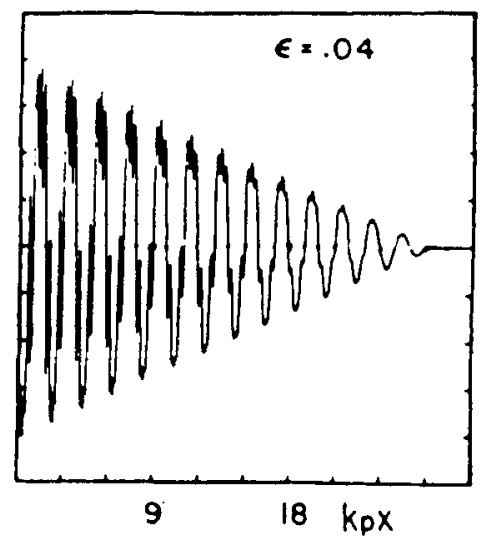

$\epsilon=.04$

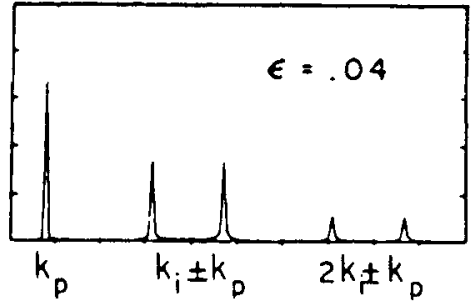

Fig. 2. Numerical solution to the Eulerian equation for the plasma wave electric field, (a) with the ripple, (b) without the ripple.

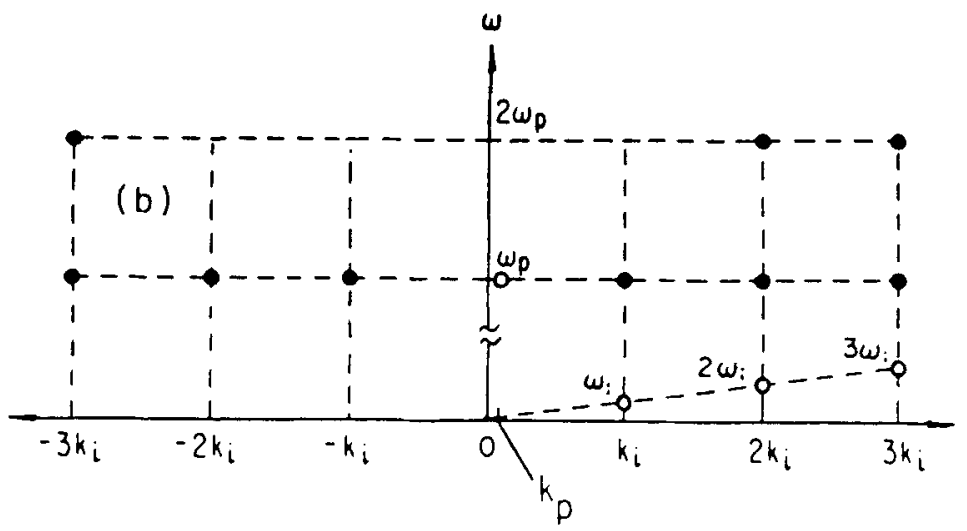

Fig. 3. Experimentally observed ion and plasma wave modes using ruby laser Thomson scatter diagnostic. The mode marked $\left(\omega_{p}, k_{p}\right)$ is the beat driven plasma wave. 
Laser to plasma wave coupling efficiency. Another key topic we have been interested in is the laser to plasma wave coupling efficiency. One of the crucial questions in determining this fraction and comparing with a theoretical model is the coupling between the electromagnetic waves and the plasma wave when $\Delta \omega=2 \omega_{\mathrm{p}}$. In other words when the beat frequency is equal to twice the plasma frequency. In the PBWA this coupling occurs because the frequency difference between the first Stokes/anti-Stokes and the high/low frequency pump is $2 \omega_{\mathrm{p}}$. Unfortunately, there is no direct experimental way to determine if such coupling is important. We therefore carried out some preliminary experiments where $\Delta \omega=\omega_{\mathrm{p}}$ coupling was not present and the only possible coupling was $\Delta \omega=2 \omega_{\mathrm{p}}$

Using the same two lines of the $\mathrm{CO}_{2}$ laser as before $(9.6 \mu \mathrm{m}$ and $10.6 \mu \mathrm{m}$, approximately) but one-fourth the resonant density we looked for $\Delta \omega=2 \omega_{\mathrm{p}}$ coupling using Thomson scatter as a diagnostic. The plasma wave from such coupling at $\mathrm{k}_{\mathrm{p}}=\omega_{\mathrm{p}} / \mathrm{c}$ mode couples with the ion wave due to SBS to give a characteristic scattering signal at $\omega_{p}$ and $k_{i}$ $\cong 2 \mathrm{k}_{\mathrm{o}}$ or at roughly $7^{\circ}$ scatter angle.

Figure 4 shows the results of this experiment. Initially only a single frequency $\mathrm{CO}_{2}$ beam $(10.6 \mu \mathrm{m})$ was focused into the plasma. The Thomson scattered ruby light (Fig. 4a) at $7^{\circ}$ showed the two usual characteristic peaks. The (essentially frequency unshifted) peak due to SBS ion wave and a small peak frequency shifted by $\omega_{\mathrm{p}}$ due to Raman scattering. The density of the plasma was adjusted until $2 \omega_{p}=\Delta \omega$. At this point the beating lasers were sent into the plasma. Figure $4 \mathrm{~b}$ shows the response of the plasma. We see the SBS peak and a smaller peak at the beat frequency. But more spectacular is the enhancement observed to the signal at half the beat frequency. We also have a smaller peak at $-\omega_{p}$ confirming that mode coupling was taking place. This result shows the importance of including the $\Delta \omega=2 \omega_{\mathrm{p}}$ coupling when estimating the laser to plasma wave coupling efficiency. Further tests are being carried out especially in the forward scattered infrared spectrum to estimate the extent of this coupling.

Development of the MARS Laser. For the past twelve months we have spent a great deal of time on building a $100 \mathrm{ps}$, high power $\mathrm{CO}_{2}$ laser system: MARS. The schematic of this system is shown in Fig. 5. The basic design output characteristics of this laser are as follows:

\section{Table 1 Laser Parameters}

$\begin{array}{ll}\text { Laser wavelengths } & 9.6 \text { and } 10.3 \mu \mathrm{m} \\ \text { Energy per line } & 20 \mathrm{~J} \\ \text { Pulse length } & 50-200 \mathrm{ps} \\ \text { Focused intensity } & 10^{14} \mathrm{~W} / \mathrm{cm}^{2} \\ \text { Spot size } & 600 \mu \mathrm{m} \\ \text { Rayleigh length } & 6 \mathrm{~cm}\end{array}$

There are three main components to this laser. An oscillator is used to produce the two frequency temporally and spatially overlapping $\approx 100 \mathrm{ps}$ pulse in the $1 \mathrm{~mJ}$ energy 


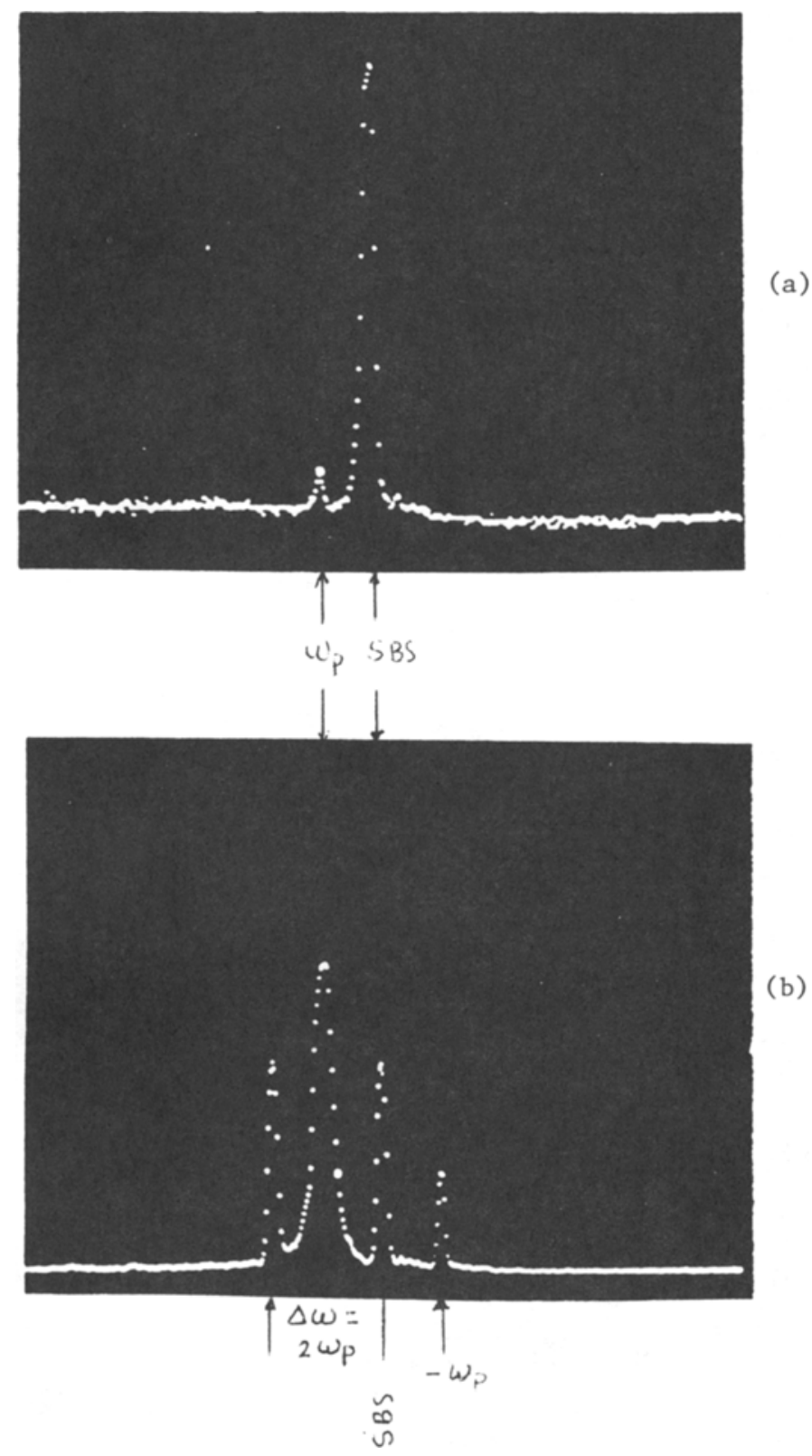

Fig. 4. Single frequency (a) and double frequency (b) excitation of plasma with density one-fourth the resonant density or the beat frequency twice the plasma frequency. 


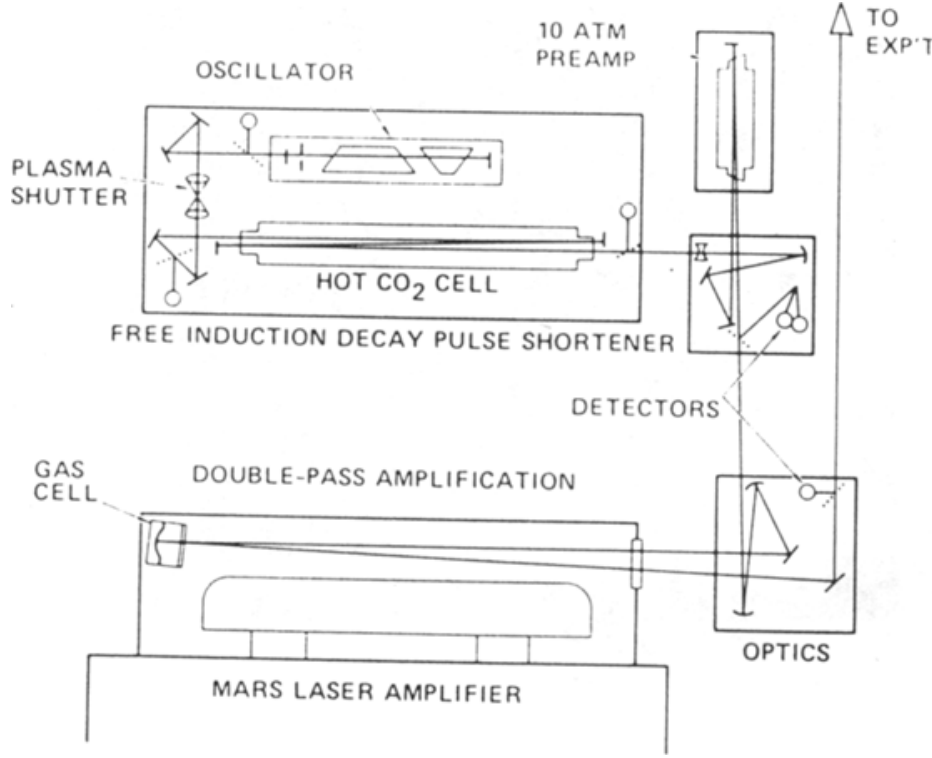

Fig. 5(a). The schematic of the MARS laser system at UCLA.

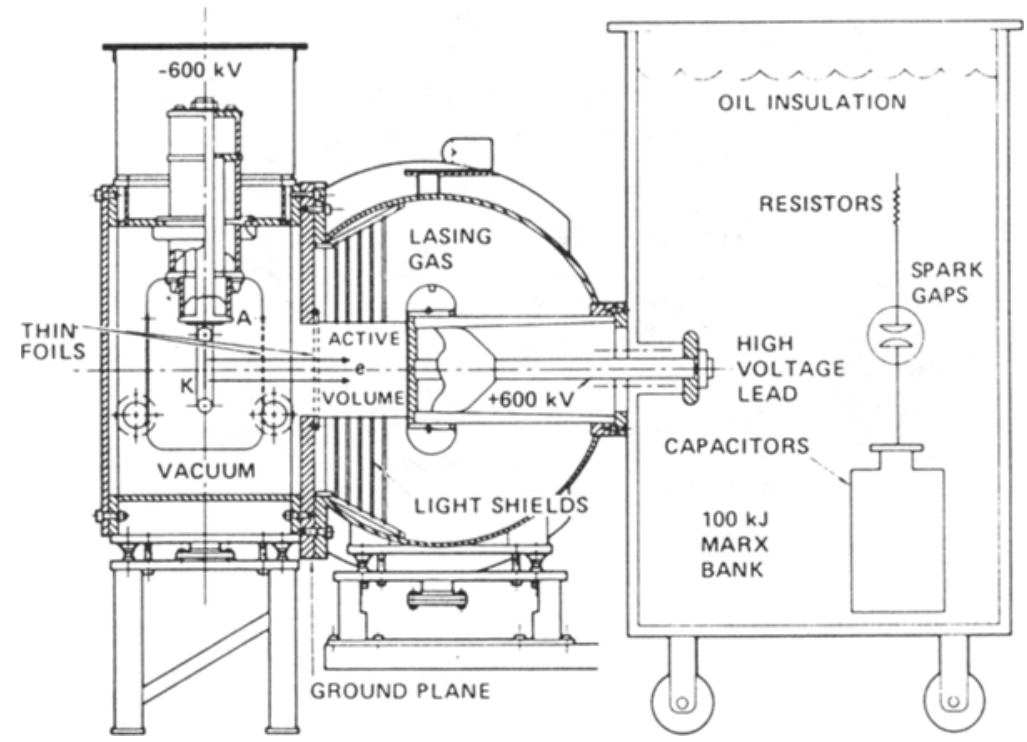

Fig. 5(b). The details of the cross-section of the E-beam pumped, $21 / 2$ atmosphere $\mathrm{CO}_{2}$ amplifier (MARS System--UCLA). 
range. A $10 \mathrm{~atm}$ preamplifier is used to amplify the pulse for injection into a $3 \mathrm{~atm}, \mathrm{E}$ beam pumped power amplifier. The oscillator uses "free induction decay" technique for generating a short pulse from a $100 \mathrm{~ns}$ long pulse from a conventional TEA module. Figure 6 shows schematically the sequence of events that lead to the generation of a subnanosecond pulse. A 100 ns long pulse is switched off by an overdense spark in an optical shutter within few tens of picoseconds at the peak of the pulse. This transmitted pulse is subsequently absorbed in a 2 meter long, hot $\mathrm{CO}_{2}$ cell. The temperature of the hot $\mathrm{CO}_{2}$ is maintained at $400{ }^{\circ} \mathrm{C}$ and the pressure is maintained at 40 Torr. The gas pressure inside the hot cell determines the collision time which can be as low as 30 picoseconds and thus determines the output pulse duration. At our working gas pressure we have already obtained detector limited pulse duration of 400 ps (10\%-90\%). The theoretically expected pulse duration is about 200 ps at this pressure. We have yet to show that this technique will work for two frequencies of interest, $9.6 \mu \mathrm{m}$ and $10.3 \mu \mathrm{m}$, but we foresee no fundamental difficulty.

The preamplifier (Lumonics 280 ) and the E-beam pumped main amplifier are in place and gain tested. The small signal single pass gain of the E-beam pumped amplifier at 2 atm pressure $\left(80 \% \mathrm{CO}_{2}, 20 \% \mathrm{~N}_{2}\right)$ was measured to be $\alpha \mathrm{L}=6$. The laser system should be integrated within the next few months and be operational by the end of the year.

Plasma source. In our previous experiments we used a rail gap plasma source for the excitation of the plasma wave. The proposed electron acceleration experiment requires a much longer and a more homogeneous plasma than produced with a rail gap source. We are therefore developing a $\theta$-pinch source which should produce a $20 \mathrm{~cm}$ long plasma column of $\approx 1.5 \mathrm{~cm}$ diameter. The plasma density over the center $2-3 \mathrm{~cm}$ plasma length is expected to be within a few percent of the desired density. By splitting the coil of the $\theta$ pinch at the center, it is possible to create a density depression at the center and at the same time have access for radial probing of the plasma using optical diagnostics (interferometry, Thomson scattering, etc.).

Proposed electron acceleration experiment. The parameters of the proposed electron acceleration experiment are shown in Table 2. These parameters assume a 200 ps risetime pulse. As an injection source we are planning to use a $1.5 \mathrm{MeV}, 9 \mathrm{GHz}$ linac. The parameters of the injector are shown in Table 3.

Since the electron pulses from the linac have a separation of about $110 \mathrm{ps}$ and the laser pulse duration is $200 \mathrm{ps}$, the synchronization of the two will initially be by chance. We have built a $180^{\circ}$ magnetic spectrometer with either surface barrier detectors or scintillator + photomultiplier detectors to detect the accelerated electrons.

The integration of the experiment with the laser and the diagnostics is expected to take place in the Fall of 1986 and preliminary experiments will be carried out in the Winter and Spring of 1987. 

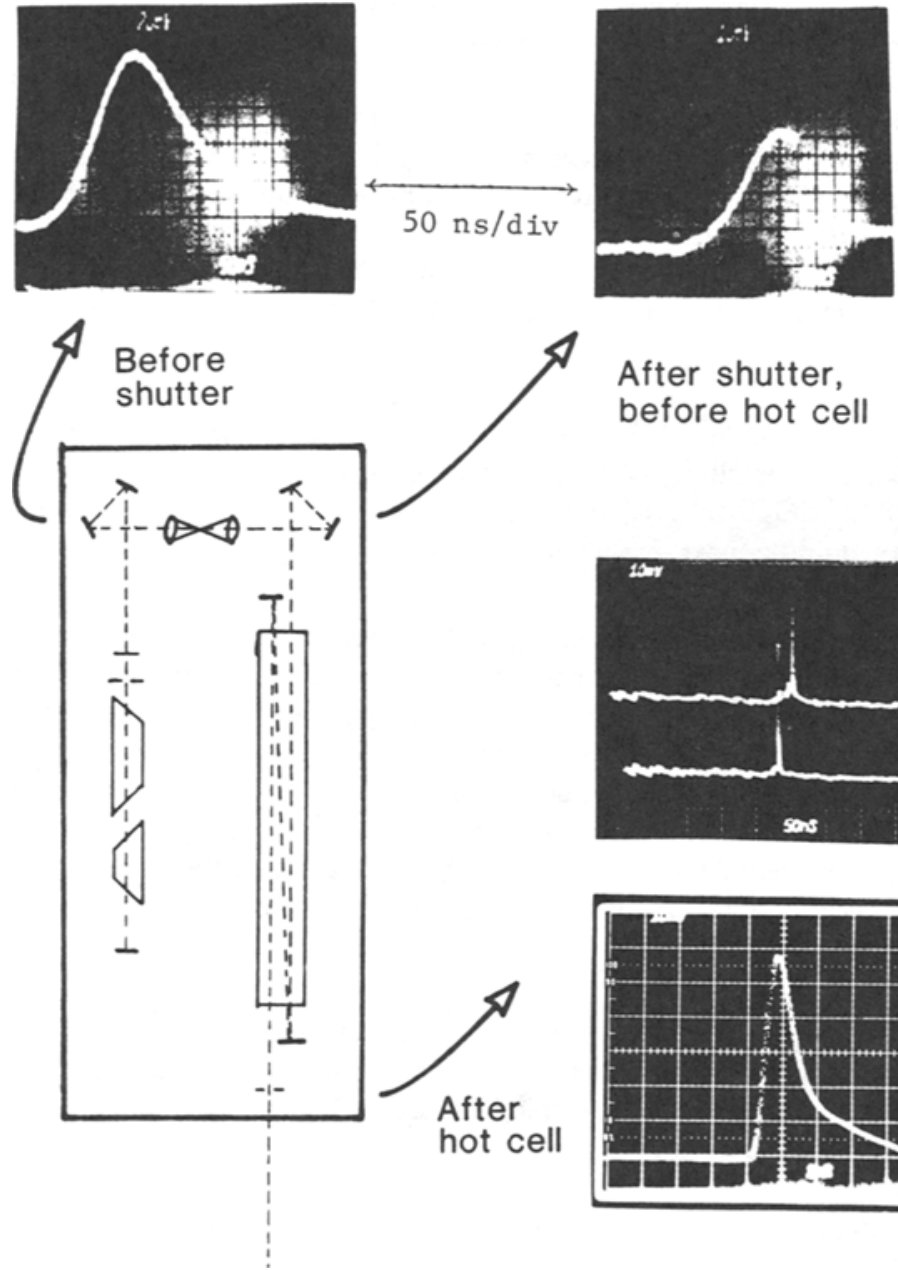

After shutter, before hot cell

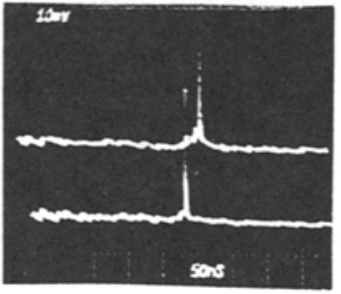

After

hot cell

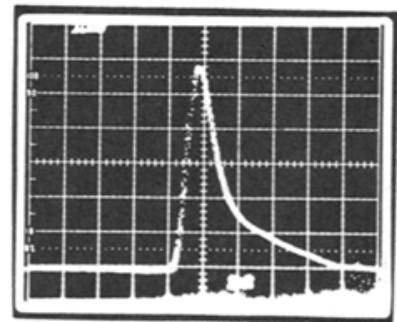

$\leftarrow 400 \mathrm{ps}$

detection

limited

Fig. 6. Picosecond $\mathrm{CO}_{2}$ oscillator based on the free induction decay (FID) principle at UCLA. 
Table 2 Laser wavelengths

Laser wavelengths

$\lambda_{0}$

$\lambda_{1}$

Resonant density $\left(n_{0}\right)$

$\alpha_{0}, \alpha_{1}$

Phase velocity of wave $\left(\gamma_{\phi}\right)$

$\mathbf{n}_{\mathbf{1}} / \mathbf{n}_{\mathbf{o}}=\boldsymbol{\varepsilon}$ expected maximum

Saturation time

Accelerating field $\left(\mathrm{eE}_{\mathrm{L}}\right)$

$$
\text { expected }
$$

maximum

Acceleration length

Injection energy

minimum

maximum

Energy gain

expected

maximum
$9.56 \mu \mathrm{m}$

$10.27 \mu \mathrm{m}$

$5.8 \times 10^{16}\left(\mathrm{~cm}^{-3}\right)$

0.07

14

0.04

0.19

113 ps

$1 \mathrm{GeV} / \mathrm{m}$

$4.75 \mathrm{GeV} / \mathrm{m}$

$1.7 \mathrm{~cm}$

$500 \mathrm{keV}$

$2.4 \mathrm{MeV}$

$16 \mathrm{MeV}$

$47.4 \mathrm{MeV}$

Table 3 Injector parameters of the UCLA experiment

Energy

No. of particles per bunch

Emittance

Micropulse duration

Pulse separation

$\Delta \mathbf{P} / \mathbf{P}$

$\sigma_{\mathrm{x}}$
$1.5 \mathrm{MeV}$

$2 \times 10^{7}$

$2 \pi \times 10^{-6} \mathrm{~mm} \cdot \mathrm{mrad}$

$10 \mathrm{ps}$

$\approx 110 \mathrm{ps}$

$10 \%$

$1 \mathrm{~mm}$ 


\section{THEORY PROGRAM}

Theoretical work at UCLA progresses along two fronts. The first is in direct support of the upcoming experiments and the second is in the exploration of advanced topics for a future plasma accelerator device. Under the first category we will present calculations on the nonlinear beat wave frequency shift and on radial fields in the UCLA experiment. Under the second category we will present work on beam loading, a new "moving" simulation code and on plasma wakefields.

Resolution of a beat wave frequency shift controversy. Prior to the Malibu Workshop ${ }^{(4)}$ we understood the growth of the plasma beat wave as that of a resonantly laser driven harmonic oscillator of natural frequency $\omega_{\mathrm{p}}$. Saturation was caused by relativistic detuning when the oscillator became relativistically heavy, thereby lowering its plasma frequency. This behavior was well described by a Lagrangian fluid model ${ }^{(5)}$. At the Malibu conference it was suggested that an Eulerian fluid description would give a positive frequency shift. Namely,

$$
\begin{gathered}
\omega_{\text {LAGRANGE }}=\omega_{\mathrm{p}}\left(1-\frac{3}{16} \varepsilon^{2}\right) \\
\omega_{\text {EULER }}=\omega_{\mathrm{p}}\left(1+\frac{5}{16} \varepsilon^{2}\right)
\end{gathered}
$$

where $\varepsilon=\mathrm{eE} / \mathrm{m} \omega_{\mathrm{p}} \mathrm{c}$ is the normalized plasma wave amplitude.

This discrepancy has now been resolved by W. B. Mori and independently by McKinstrie and Forslund ${ }^{(5)}$. The reader is referred to their works as only a superficial summary is given here. The problem was revealed to be in the expansion of the current $j$ to second order in the Eulerian model:

$$
\begin{gathered}
\mathrm{j}_{2} \approx \mathrm{n}_{1} \mathrm{v}_{1}+\mathrm{n}_{\mathrm{o}} \mathrm{v}_{2} \\
\mathrm{n}_{1} \propto \cos \chi, \quad \mathrm{v}_{1} \propto \cos \chi, \quad \mathrm{v}_{2} \propto \cos 2 \chi
\end{gathered}
$$

where $\mathrm{n}_{\mathrm{o}}$ is the constant plasma density, subscripts refer to the order of the density and velocity and $\chi$ is the phase $k_{p} x-\omega_{p} t$. Thus the time averaged $j_{2}$ is $\left\langle j_{2}\right\rangle \propto\left\langle\cos ^{2} \chi\right\rangle-1 / 2$ $\neq 0$. But Ampere's Law gives

$$
(\vec{\nabla} \times \vec{B})_{x}=4 \frac{\pi}{c} j+\frac{1}{c} \dot{E}=0
$$

so that $\left\langle\mathrm{j}_{2}\right\rangle$ implies $\langle\dot{\mathrm{E}}\rangle \neq 0$. In a real plasma DC E-field would grow (this is the socalled " $k=0$ mode") to stop $<j>$. The remarkable paradox is that in order to allow the average current $\left\langle\mathrm{j}_{2}\right\rangle$ to be zero we must allow the second order velocity to have a drift $\left\langle v_{2}\right\rangle \neq 0$ ! When $\left\langle v_{2}\right\rangle$ is solved for self-consistently one finds that the resulting Doppler 
shift gives

$$
\omega_{\text {EULER }} \rightarrow \omega_{\text {EULER }}-\mathrm{k}_{\mathrm{p}}\left\langle\mathrm{v}_{2}\right\rangle=\omega_{\text {LAGRANGE }} .
$$

Thus the two descriptions agree. The importance of the negative frequency shift, as reported by Tang and Sprangle ${ }^{(6)}$ at Malibu, is that larger plasma wave amplitudes (order $50 \%$ larger) can be obtained if the lasers' beat frequency $\Delta \omega$ is slightly below $\omega_{\mathrm{p}}$. In other words, the plasma density in our experiment should be tuned to a value slightly higher than the resonant density where $\omega_{p}=\Delta$. Another consequence of the negative frequency shift is that the wave is eventually subject to a modulational instability.

Radial fields. Here we analyze the importance of radial plasma wave fields on test particle acceleration in the upcoming experiments. A physical picture of how radial fields arise is given in Fig. 7. Radial fields have been found previously for a given plasma wave profile by Lawson, et al. ${ }^{(7)}$ and for a given wakefield driver by Ruth, et al. ${ }^{(8)}$. More relevant to our beat wave experiment, we ask what are the radial profiles of the radial field $E_{r}(r)$, accelerating field $E_{z}(r)$ and plasma density perturbation $n_{1}(r)$ for a given gaussian laser profile:

$$
E_{\text {LASERS }}=E_{1,2} e^{-r^{2} / R^{2}} \cos \left(k_{p} z-\omega_{p} t\right) \Theta(t-z / c)
$$

This calculation has now been published in ref. 9 and we merely summarize the results graphically in Fig. 8. The parameters of Fig. 8a are similar to those of the INRS experiments ${ }^{(10)}$ while Fig. $8 \mathrm{~b}$ corresponds to those at $\mathrm{UCLA}^{(4)}$. The radial fields are zero on axis and become quite large off axis. The $\mathrm{E}_{\mathbf{z}}$-profile follows that of the $\mathrm{E}_{\text {LASERS; the normal- }}^{2}$ ized plasma density perturbation does not. This difference can be important to Thomson scattering diagnostics in which $n_{1}$ is measured and $E_{2}$ is inferred from $n_{1}$.

Having solved for the fields we can now examine test particle trajectories numerically. Sample trajectories are shown in Fig. 9 for particles at various initial radii and phases. At each radius three particles were injected in phases of the wave corresponding to focusing, defocusing and null focusing; all were in the accelerating phase (see Fig. 7). Particles on or near the axis $(r<20 \mu)$ exhibit the expected behavior (including betatron motion), but some particles at larger radii (and larger radial fields) are detrapped (or at least deflected away with little energy gain) even though they have the proper phase to be focused and accelerated.

One way in which particles can detrap is by acquiring a radial velocity component. In an experiment this might be the result of radial fields or merely of the emittance of the injected beam. Then the particles parallel velocity may drop (since $v_{\text {TOTAL }}<c$ ) to the point that they fail to keep up with the high velocity wave. We have calculated analytically the minimum initial energy an electron must have to be trapped by a wave of given amplitude for various angles $(\theta)$ that the particle makes critically relative to the wave (Fig. 10). 


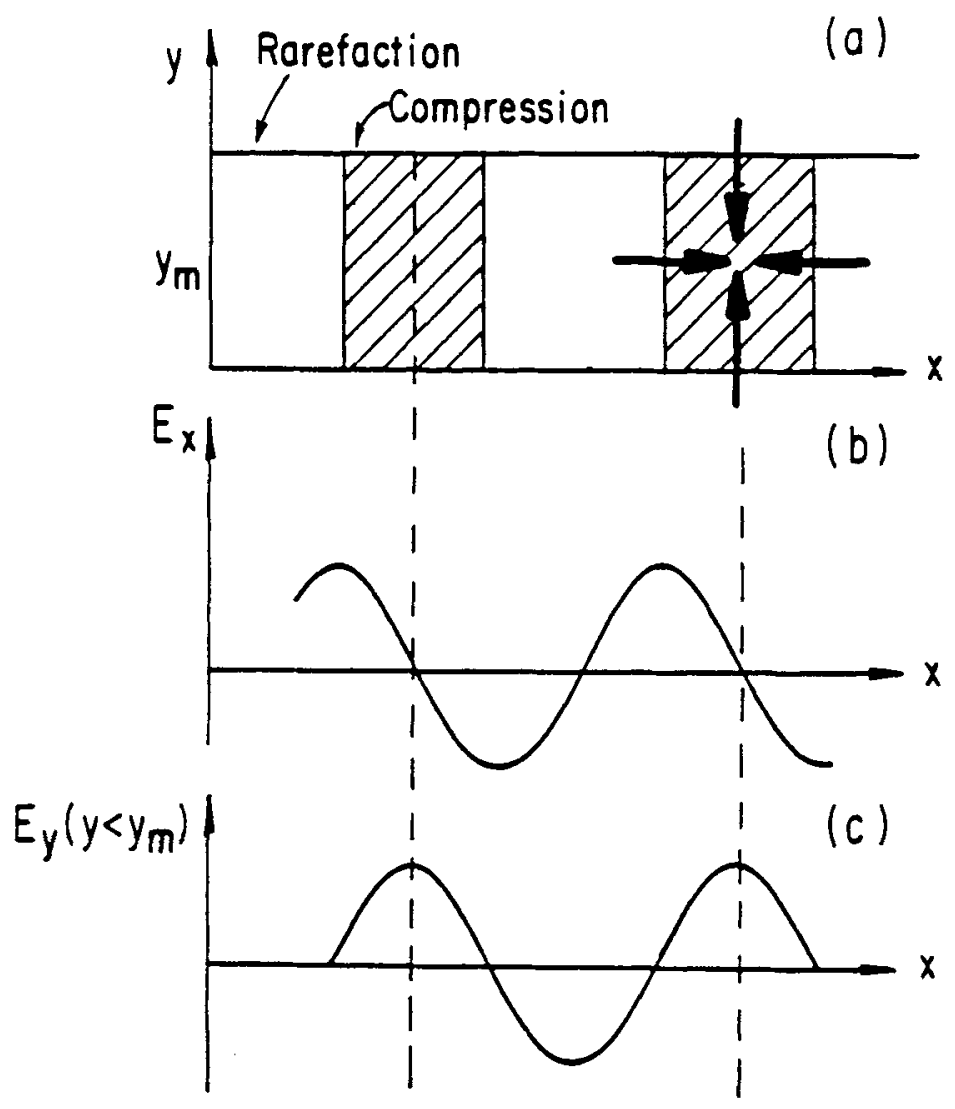

Fig. 7. Physical picture of how radial fields arise in a plasma wave (a) and the longitudinal variation of the accelerating field (b) and the radial field (c). 


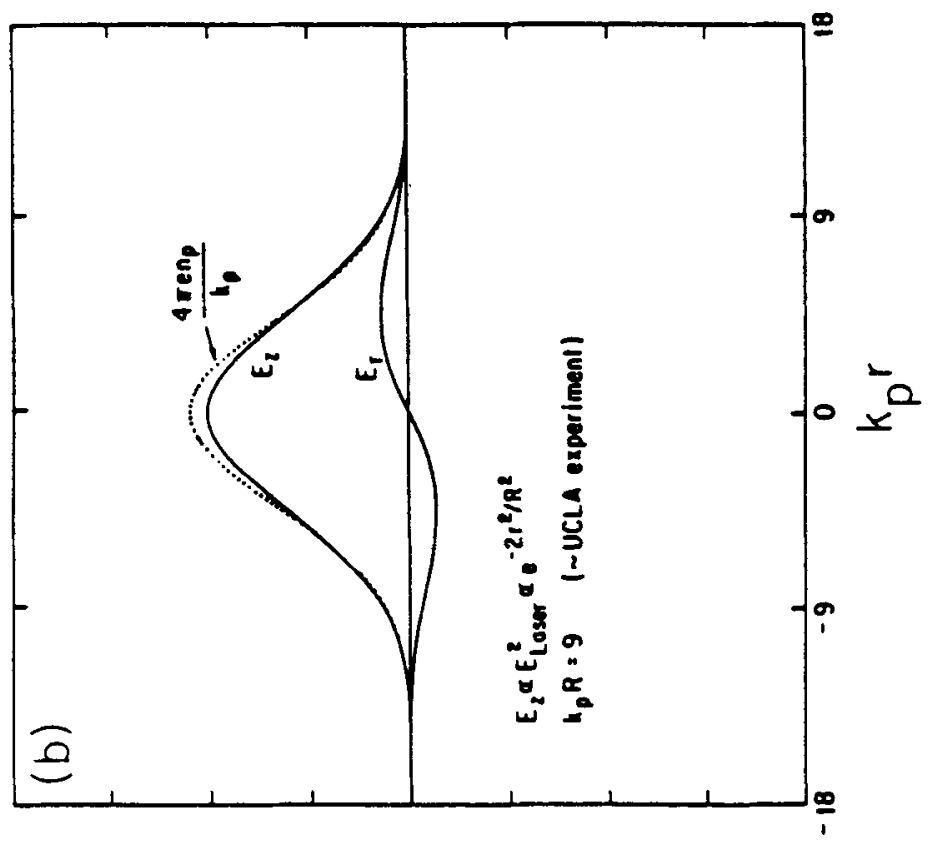

苟

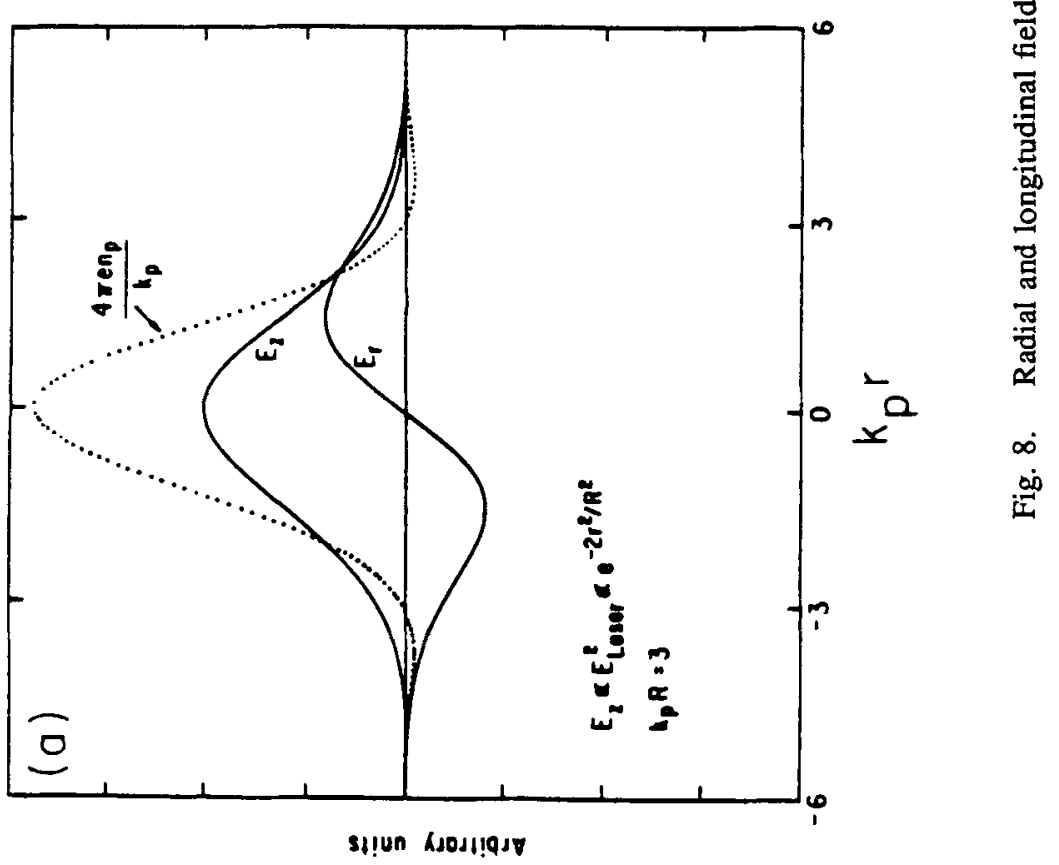




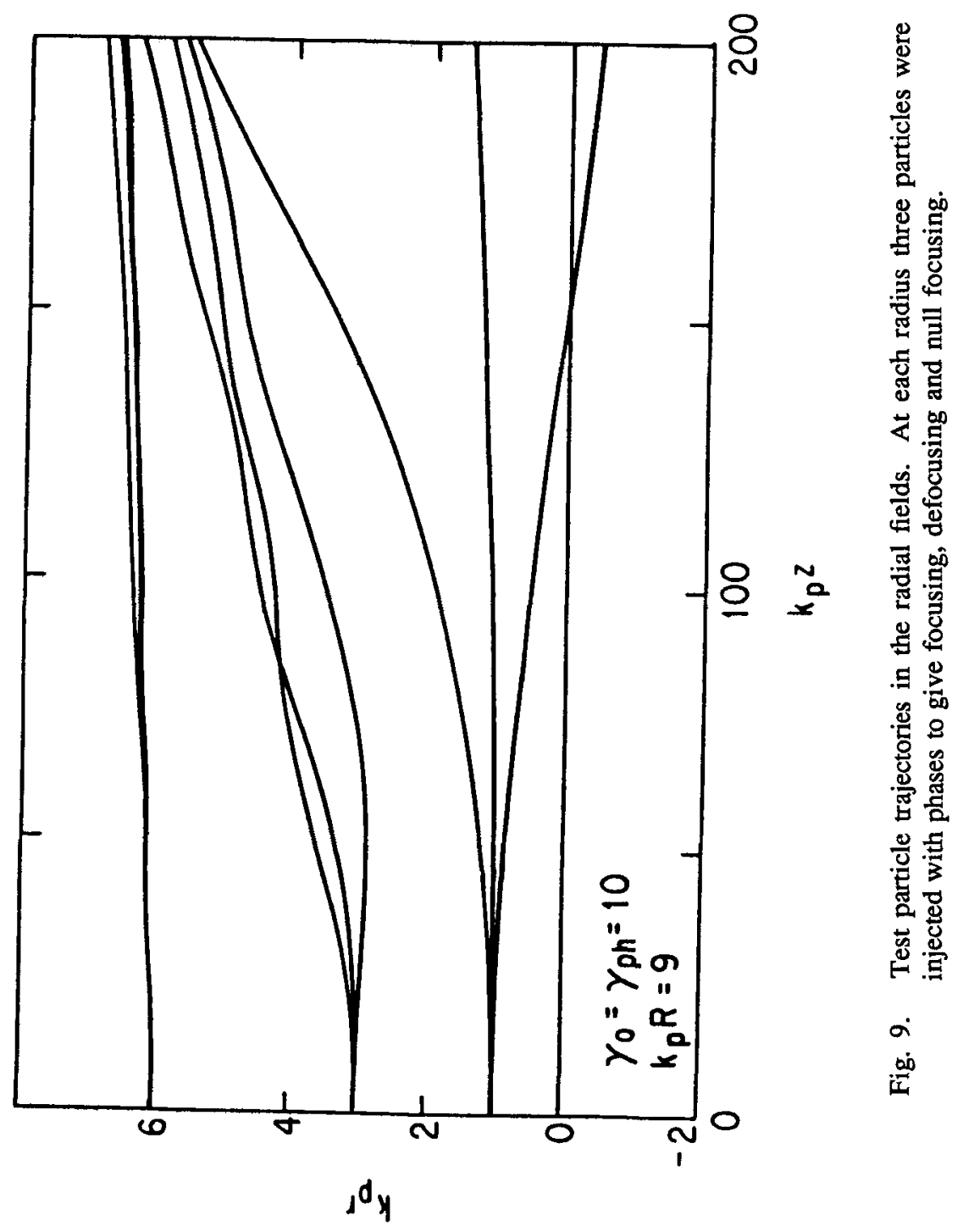




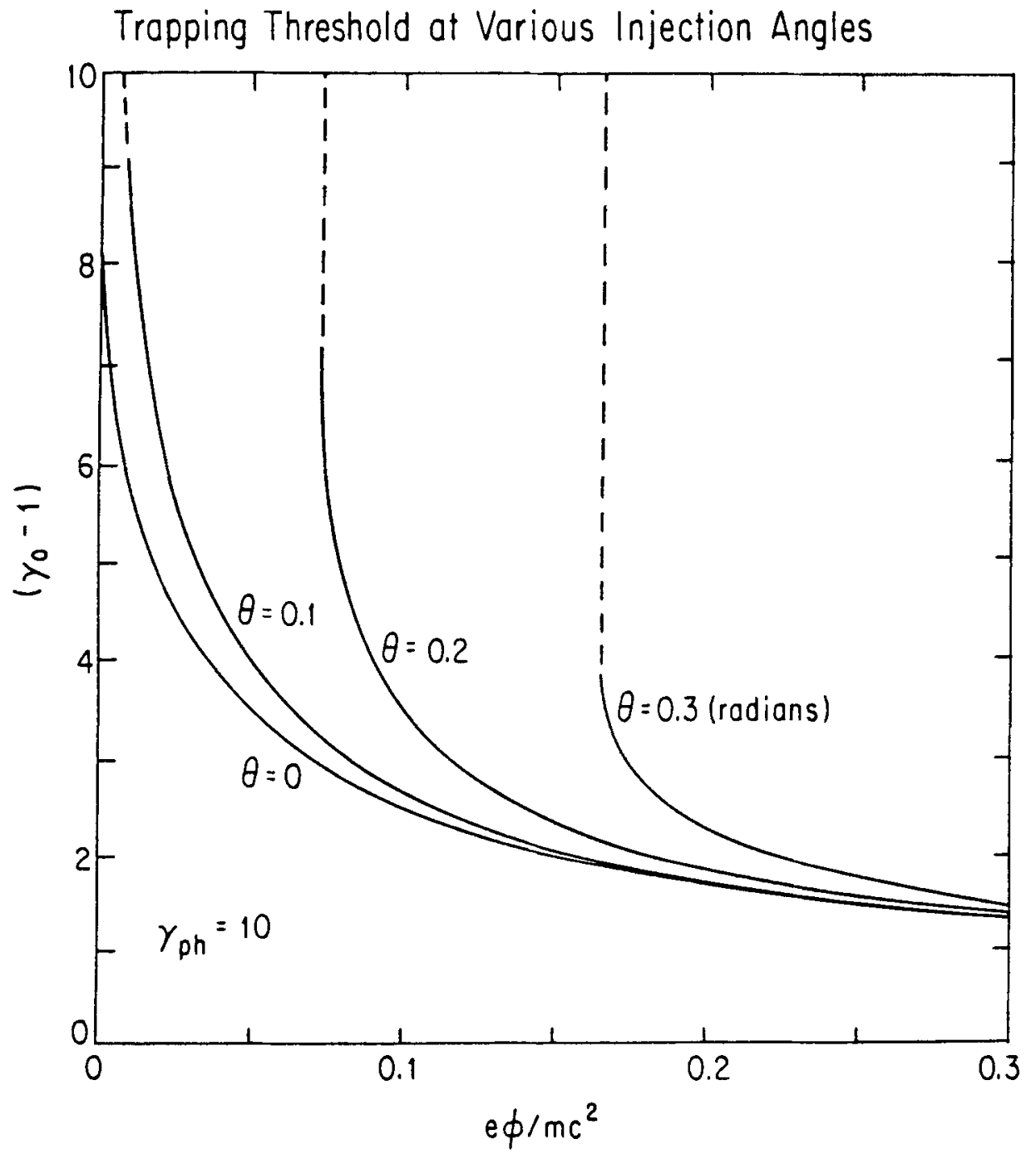

Fig. 10. Trapping threshold vs plasma wave amplitude for particles making various angles with respect to the axis of the wave. 
We find that for the parameters typical of UCLA experiments the injection threshold for particle acceleration and trapping is $-1.5 \mathrm{MeV}$ for particles making an angle less than $5^{\circ}$ relative to the wave. For injection angles $\theta>10^{\circ}$, no particles can be trapped in a wave of normalized amplitude $e \phi / \mathrm{mc}^{2}<0.1$.

We can estimate the number of particles that will be accelerated in our experiment as follows:

No. of injected electrons $\left(100 \mathrm{~mA} \times 10 \mathrm{psec}\right.$ in $\left.1 \mathrm{~mm}^{2} \mathrm{spot}\right) \approx 10^{7}$

No. accelerated $=10^{7} \times \frac{\pi(20 \mu)^{2}}{(1 \mathrm{~mm})^{2}} \times \frac{1}{4} \approx 10^{3}$

The factor of $1 / 4$ arises because only $1 / 4$ of the random phases are both accelerating and focusing and the area factor is based on the numerical solutions (Fig. 9). This number is easily detectable. We note that the above analysis assumes particle injection in the linear beat wave regime ( $\sim$ first $100 \mathrm{psec}$ ) before saturation. After saturation the radial profiles are drastically modified. Test particle trajectories in this case are examined in the computer simulation section which follows.

Beam Loading. We have recently begun to address issues associated with beam loading in plasma waves ${ }^{(1)}$. We consider a cold plasma wave which has the form

$$
\vec{E}_{w}=4 \pi e \frac{n_{1}}{k_{p}} \cos \omega_{p}(t-z / c+\phi) \hat{z}
$$

where $\phi$ is an arbitrary phase constant. This wave could be created by beat wave, wakefield or other means, and the analysis which follows applies to all plasma wave schemes. For such a plasma wave we ask what is the maximum number of particles $\left(\mathrm{N}^{\max }\right)$ the wave can accelerate, what is the luminosity $(\mathrm{L})$, what is the energy spread $(\Delta \gamma / \gamma)$, what is the emittance $(\varepsilon)$ and what is the efficiency $\left(\eta_{b}\right)$ with which wave energy is extracted by the particles?

Fortunately, the formalism developed for studying wakefields provides us a tool for attacking these questions. The effect of loaded beam particles on the wave can be found by superposing the wakefields of the beam particles on the wave field. The single electron wake function is

$$
E_{1}=2 \omega_{p}^{2} K_{o}\left(k_{p} r\right) \cos \left(\omega_{p}(t-z / c)\right) \Theta(t-z / c),
$$

where $\Theta$ is the step function and the electron's position is approximated by $z=c t$.

For wide beams and waves (larger than a few $c / \omega_{\mathrm{p}}$ ), we find that $100 \%$ wave absorption occurs if $\mathrm{N}^{\max }$ electrons are placed at a phase $\phi=\pi$, where $\mathrm{N}^{\max }$ is given by $\mathrm{E}_{\mathrm{W}}+\mathrm{N}^{\max } \mathrm{E}_{1}=0$ or

$$
\mathrm{N}^{\max }=\frac{\mathrm{n}_{1}}{\mathrm{k}_{\mathrm{p}}} \mathrm{A}=5 \times 10^{5} \sqrt{\mathrm{n}_{\mathrm{o}}} \mathrm{A}\left(\mathrm{cm}^{2}\right) \frac{\mathrm{n}_{1}}{\mathrm{n}_{\mathrm{o}}}
$$


and $n_{1} / n_{0}$ is given in $\mathrm{cm}^{-3}$, and $A$ is the cross-sectional area of the beams and waves. The wave absorption is illustrated dramatically by the 1-D simulation of Fig. 10 .

The use of $N^{\max }$ particles at $\phi=\pi$ gives $100 \%$ beam loading efficiency only at the expense of $100 \%$ energy spread. The reason for this is that the first particle in the bunch feels the full accelerating field while the last particle feels zero field ${ }^{(11)}$. Two possible scenarios for reducing the energy spread are depicted in Fig. 11 and 12. The first figure shows how a shaped trailing bunch density (in the form of a reverse triangle or a portion of one) gives a constant accelerating field within the bunch. Neglecting phase slippage the energy spread approaches zero. 1-D simulations of shaped beam loading are shown in Fig. 13. The trade offs between energy spread, gradient and efficiency are discussed in ref. 11 .

Another beam loading consideration is the emittance $\varepsilon$. The presence of radial plasma wave fields can lead to emittance growth of the beam. The emittance is approximately given by $\varepsilon=\pi \mathrm{r} \theta$ where $\mathrm{r}$ and $\theta$ are the radius and angular divergence of the beam. In terms of the betatron wavelength $\beta$, the emittance is approximately $\varepsilon \approx r^{2} / \beta$ where $\beta$ is given in terms of the radial force $F_{r}\left(q\left[E_{r}-B_{\theta}\right]\right.$ by $\left.\beta^{2}=\gamma \mathrm{mc}^{2} /\left(F_{r} / r\right)\right)$.

There are essentially two options to maintain a small emittance beam. One is to use very wide waves with flat radial profiles. Then the problem becomes essentially 1-D with no radial fields. For example, if $n_{0}=10^{16} \mathrm{~cm}^{-3}$ the wave radius should be larger than $0.3 \mathrm{~cm}\left(70 \mathrm{c} / \omega_{\mathrm{p}}\right)$ to maintain the exceedingly small emittance suggested by $\mathrm{B}$. Richter at Malibu (i.e., $\varepsilon<10^{-12} \mathrm{~cm}$ radians). Such wide waves may prove costly in terms of the driving energy required to produce them.

A second option for maintaining low emittance is to load a very narrow beam (because $\varepsilon \propto r^{2}$ ). Since the wakefield of such a beam extends to a distance of order $c / \omega_{p}$, no matter how narrow the beam is made, it is possible to retain a reasonable efficiency by using an accelerating wave of width $c / \omega_{p}^{(11,13)}$.

The radial profiles of the longitudinal wakefields of beams of radius (1.0$0.001) \mathrm{c} / \omega_{\mathrm{p}}$ are shown in Fig. 14. Even though the overlap in area of the beams is only $4 \times 10^{-4}$, the overlap in their field energy $\left(\int\left(E_{1}-E_{2}\right)^{2} r \mathrm{dr} / \int E_{1}^{2} r \mathrm{dr}\right)$ would be approximately $40 \%$ (suggesting a maximum $40 \%$ beam loading efficiency for beam and wave profiles similar to those in the figure).

For beams of arbitrary radius $\mathrm{r}$ we can write a general expression for the maximum number of beam particles a plasma wave can support analogous to Eq. (10):

$$
\mathrm{N}^{\max }=\frac{\mathrm{n}_{1}}{\mathrm{k}_{\mathrm{p}}} A_{\text {eff }}=5 \times 10^{5} \sqrt{\mathrm{n}_{\mathrm{o}}} A_{\text {eff }} \cdot \frac{\mathrm{n}_{1}}{\mathrm{n}_{\mathrm{o}}}
$$

where

$$
A_{\text {eff }}=\frac{\pi r^{2}}{1-k_{p} r K_{1}\left(k_{p} r\right)}, \begin{cases}\pi r^{2}=A & \left(r>c / \omega_{p}\right) \\ 0\left(c^{2} / \omega_{p}^{2}\right) & \left(r \ll c / \omega_{p}\right)\end{cases}
$$




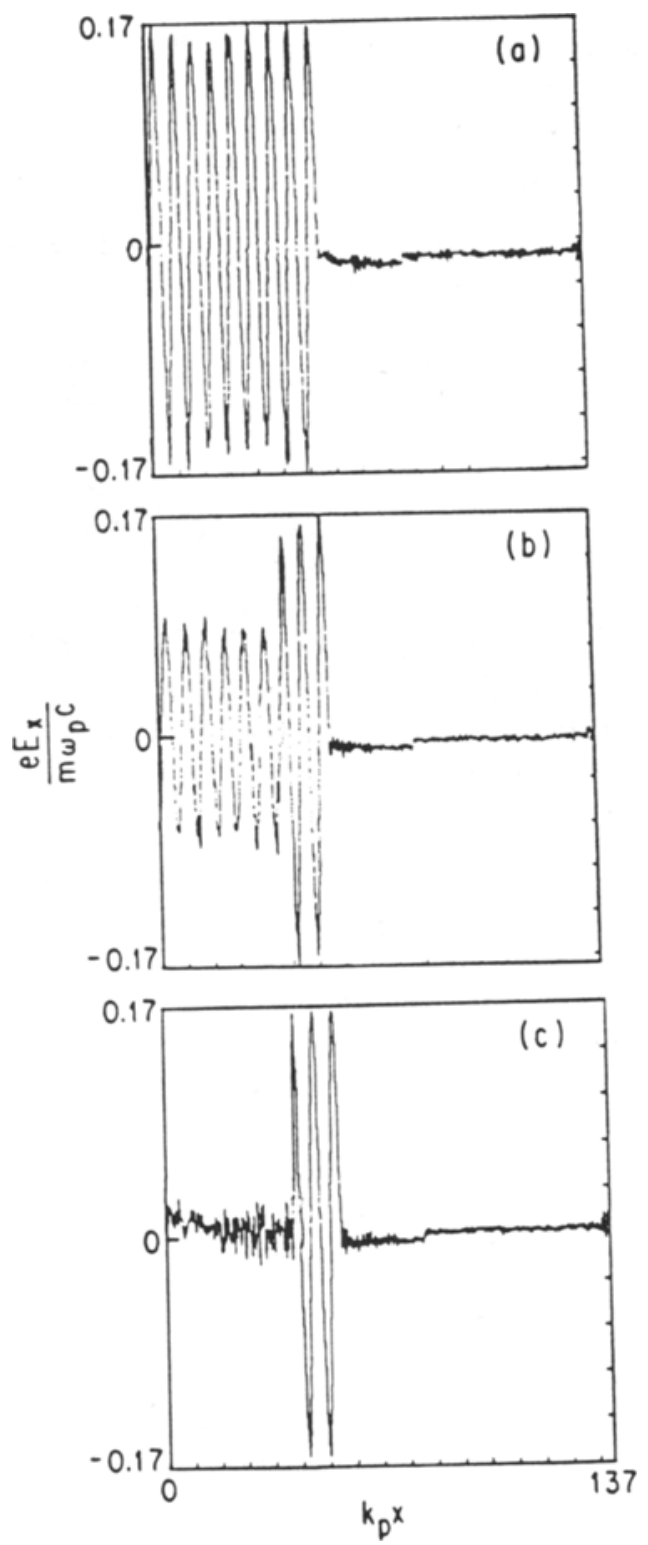

Fig. 11. One dimensional simulations of beam loading. The plasma wave is driven by a shaped electron beam (wake-field excitation) in (a). In (b) the number of injected particles $=1 / 2 \mathrm{~N}^{\max }$ and in (c) it is equal to $\mathrm{N}^{\max }$ given by Eq. (10). 


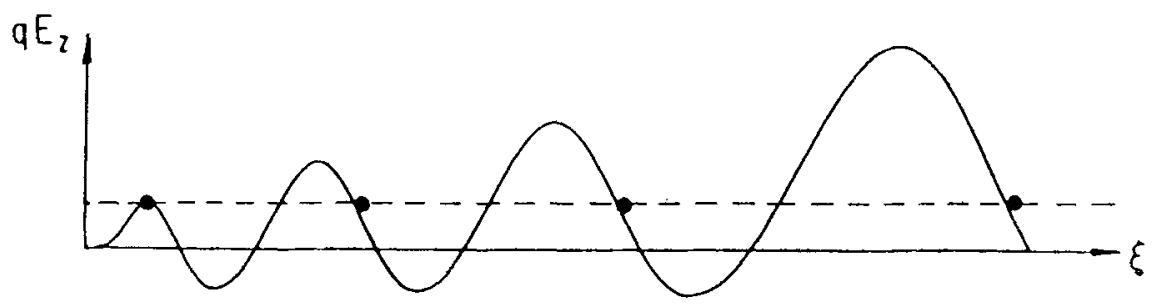

Fig. 12. One scenario for reducing the energy spread of the beam envisions placing several bunches at different phases in successive accelerating buckets so that each microbunch sees the same accelerating field.

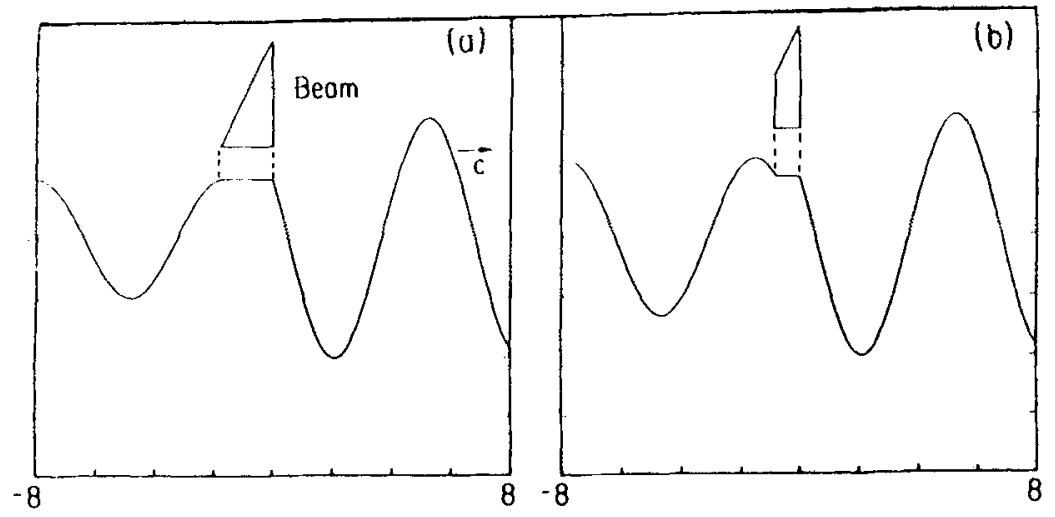

Fig. 13. A second scenario for reducing the energy spread of the accelerating bunch uses an electron bunch that is ramped down in density. 


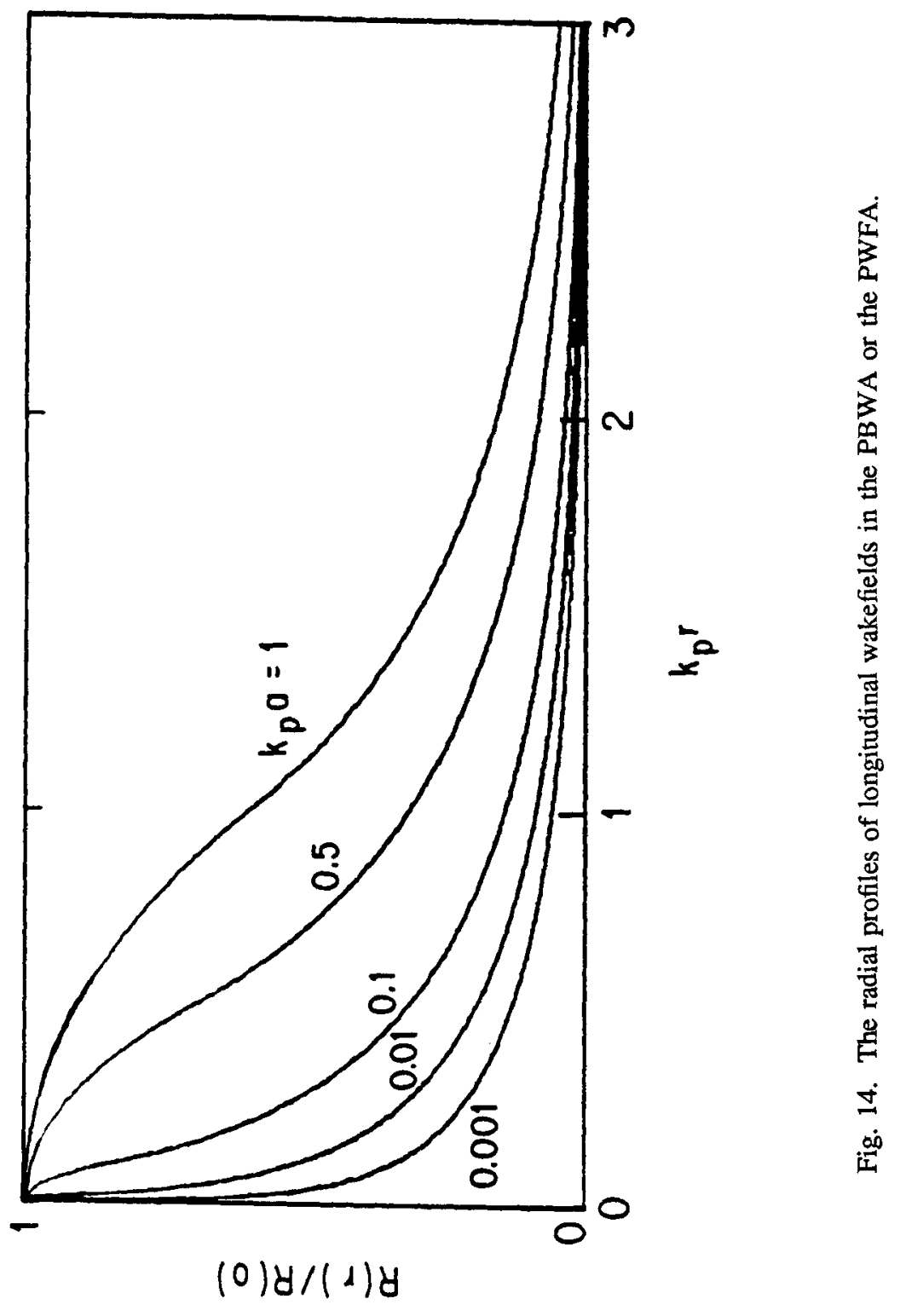


This scaling law was suggested qualitatively by R. Evans at Malibu ${ }^{(4)}$.

We consider now a beam loading example based on the non-optimized parameters suggested by $\mathrm{B}$. Richter at Malibu ${ }^{(4)}$. He assumed $\mathrm{N}=2.5 \times 10^{9}$ electrons for a $5 \mathrm{TeV}$ collider with rep rate $>1 \mathrm{kHz}$, radius at the interaction point $=10^{-2} \mu \mathrm{m}$. This gives a luminosity of $10^{34} \mathrm{~cm}^{-2} \mathrm{~s}^{-1}$. He also specified that the emittance should be less than $10^{-12} \mathrm{~cm}$.

For comparison consider the following (non-optimized) plasma example. We assume $n_{0}=10^{16} \mathrm{~cm}^{-3}, n_{1} / n_{0}=0.5$, wave width $(2 r)=2 c / \omega_{p}(=100 \mu)$, beam length is $25 \mu \mathrm{m}$ and it is shaped to give zero energy spread for particles on axis. Based on these assumptions we derive the following ${ }^{(11)}$ : The emittance requirement restricts the beam radius to be less than $0.2 \mu, \mathrm{N}=3 / 4 \mathrm{~N}^{\max }=0.3 \times 10^{9}$, electrons and the accelerating gradient is $2.5 \mathrm{GeV} / \mathrm{m}$. An energy spread of $9 \%$ results because the beam's wakefield off axis falls off faster (with $r$ ) than the wave's accelerating field does (i.e., the beam shaping is not perfect for off-axis particles).

The efficiency $\left(\eta=\int\left(E_{\text {wave }}-E_{\text {wake }}\right)^{2} r d r / \int E_{\text {wave }}^{2} r d r\right)$ is approximately $20 \%$ for this example. This non-optimized example suggests that beam loading in plasma waves is not inconsistent with the requirements for future colliders. Since there are tradeoffs between the beam loading quantities $N, \varepsilon, \nabla \gamma / \gamma$ and $\eta$, any one may be improved by relaxing the requirements on one or more of the others.

Finally, we comment on the overall efficiency of a plasma wave accelerator. The overall efficiency is given by the product of the beam loading efficiency $\left(\eta_{b}\right)$, the wall plug to driver efficiency and the driver to plasma wave efficiency. We have shown that beam loading efficiencies of $20 \%$ are possible for even very high quality beams. The wall plug to driver efficiency may be on the order of $30 \%$ for $\mathrm{e}^{-}$beam drivers and $10 \%$ for lasers at present. The driver to plasma wave efficiency has exceeded $75 \%$ in 1-D wakefield simulations and $50 \%$ in beat wave simulations (for low $\omega_{o} / \omega_{\mathrm{p}}$ ). More work is needed to predict what can be expected in more realistic examples.

Moving simulation code. A recent breakthrough in computer modelling of plasma accelerators by J. J. Su and J. M. Dawson now makes possible the simulation of more realistic energies and longer time behavior. An example of test particle acceleration from 75 $\mathrm{MeV}$ to $\sim 1 \mathrm{GeV}$ is shown in Fig. 15. This 1-D run which took 5 Cray hours would have taken 10,000 hours using a conventional plasma code. The time (and memory) savings come by "creating" new plasma in front of the moving beams and waves just before it is needed and "dropping off" the uninteresting plasma that is more than a specified distance behind. This technique is being adapted now to 2-D codes as well and will enable us to model such problems as the long time evolution (i.e., self-focusing, cascading, pump depletion) of a laser pulse.

Selected wakefield results. Although presented in greater detail in a later session (see paper by Jones et al., these proceedings), we present here some selected results on plasma wakefields. In Fig. 16 we compare the results of 1-D and 2-D simulations of the plasma wakefield accelerator ${ }^{(14)}$. At this early time the results are nearly identical. How- 


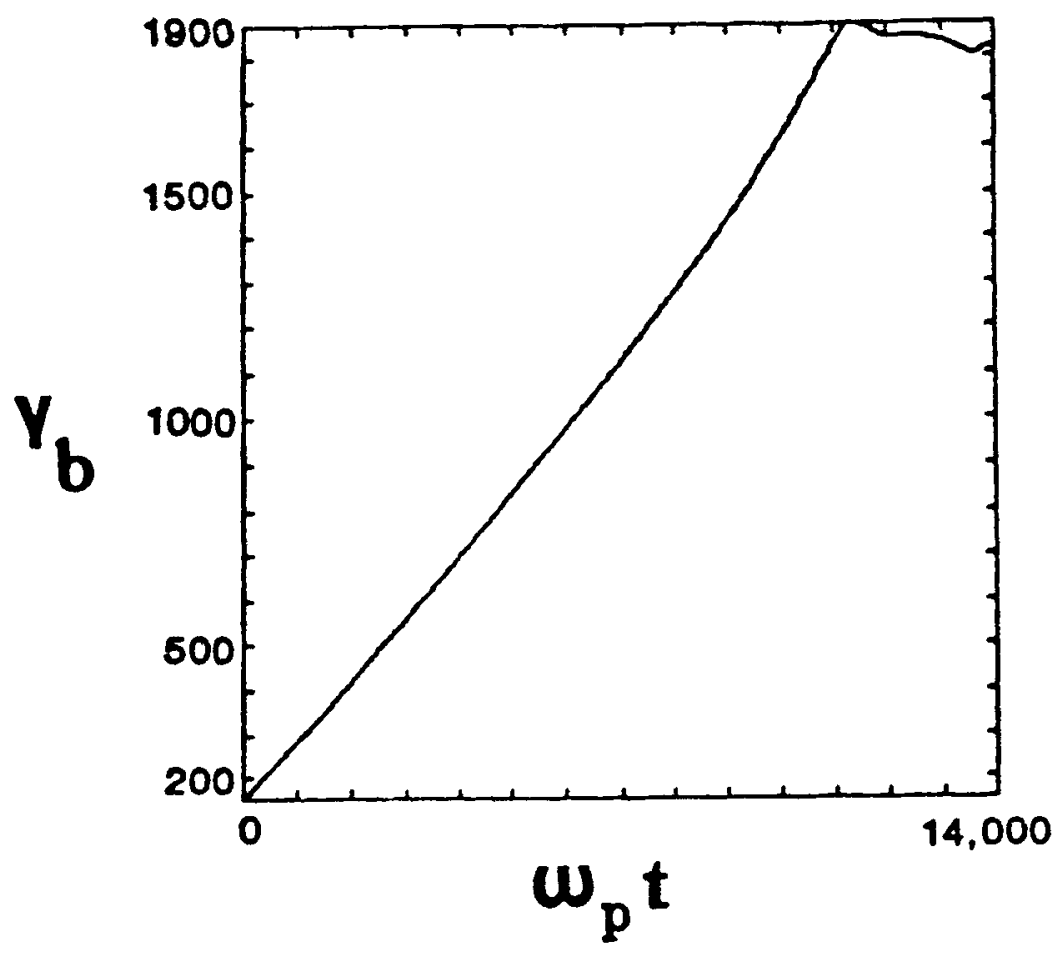

Fig. 15. Energy gain of a trailing particle in the PWFA using a $1 \mathrm{D}$, moving code simulation. 

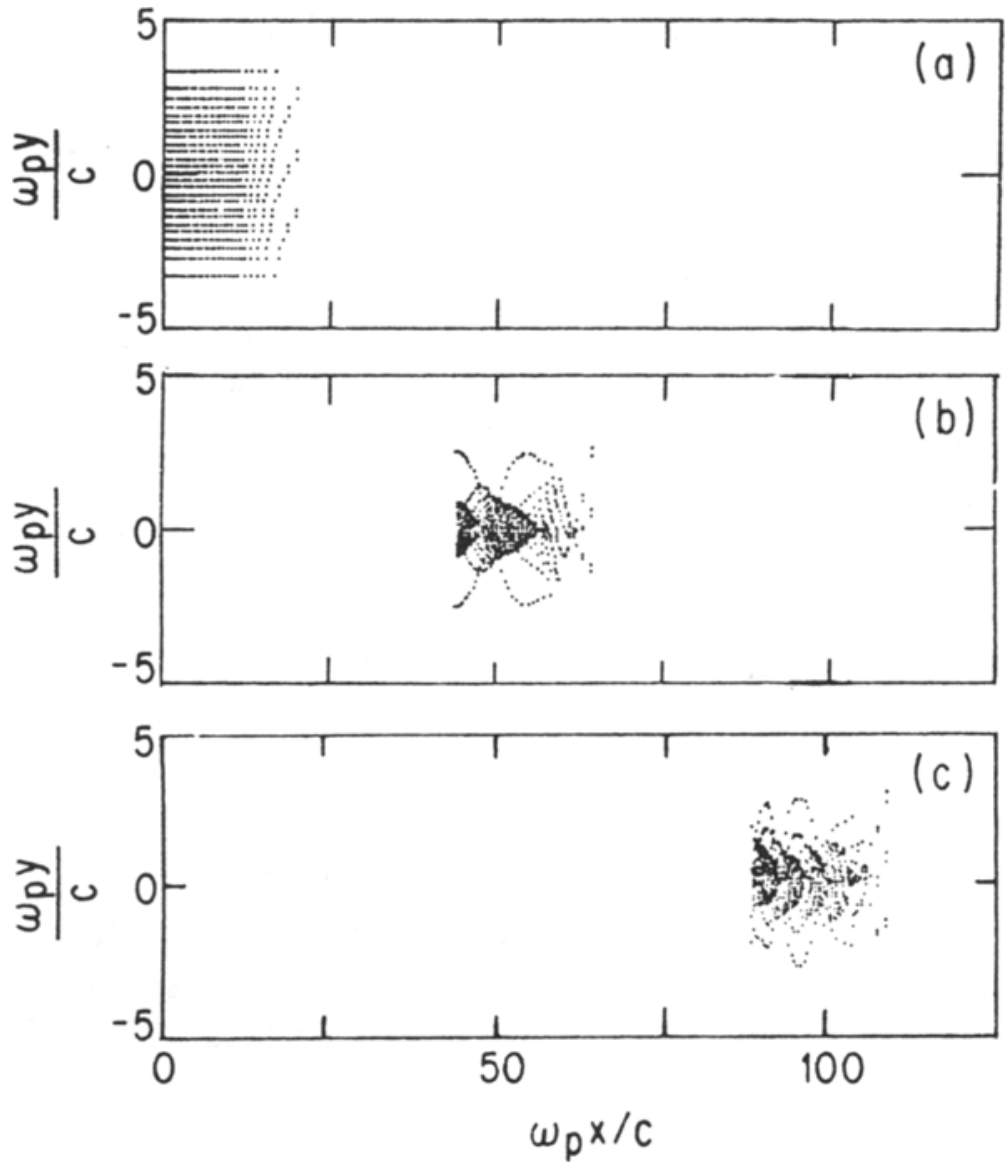

Fig. 16. Selected 2D wakefield simulation results. Severe self-pinching of the driver beam can be seen in (b). Eventually the beam comes to a new equilibrium state as shown in (c). 
ever, at later times the driving beam may be severely self-pinched as illustrated in Fig. 16(b). Eventually (Fig. 16c) the beam appears to reach a new equilibrium state. Su, Jones and Koenigs (these proceedings) have found that stable beam propagation can be obtained by preparing the initial beam with sufficient thermal energy and applying an axial magnetic field.

The self-pinching is taken to advantage in the plasma lens concept illustrated in Fig. 17. The short precursor beam provides a wake field which when superposed with the main beam's wake gives uniform pinching of the main beam initially (see P. Chen in these proceedings). However, the precursor does not pinch in parallel with the main beam, so at later times this "thick" plasma lens get a bit "fishy".

Figure 18 illustrates a means to phase lock particles in the plasma wakefield accelerator. By slightly ramping the plasma density, the local plasma frequency $\omega_{\mathrm{p}}$ increases and the plasma wavelength $\left(\lambda_{p}=2 \pi v_{b} / \omega_{p}\right)$ decreases. This causes the phase fronts to advance on the driving beam. The appropriate gradient to keep a particle of speed $\approx c$ on say the $w^{\text {th }}$ plasma wave peak would be $\mathrm{b}^{(14)} \frac{1}{\mathrm{n}} \mathrm{dn} / \mathrm{dx}=1 / \mathrm{w} \lambda_{\mathrm{p}} \gamma_{\mathrm{b}}^{2}$ where $\gamma_{\mathrm{b}}$ is the driving beam energy. The use of a phase locking technique such as this may enable the use of low energy driving beams (or even proton drivers). Also the use of shaped beams to eliminate energy spread will only work if the beams do not phase slip appreciably.

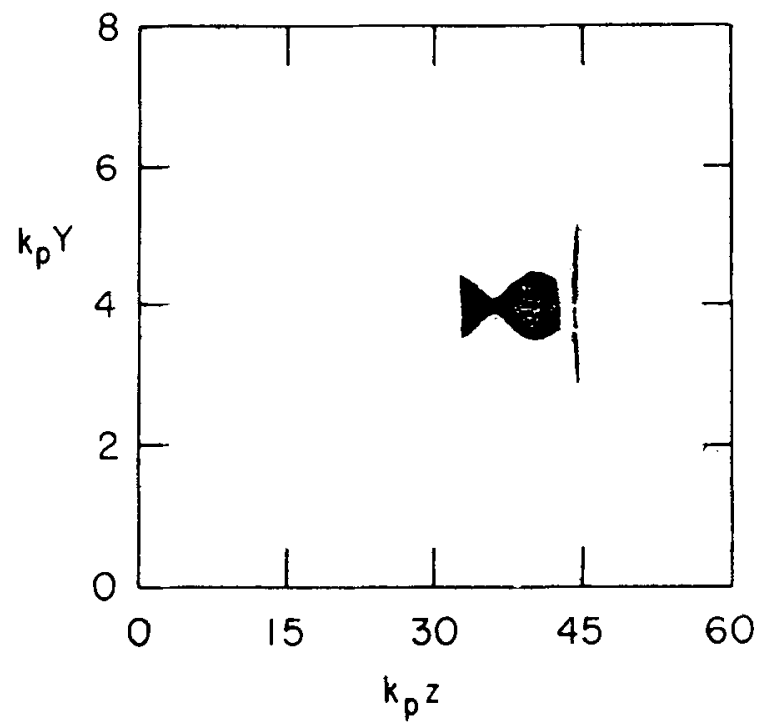

Fig. 17. Two dimensional particle simulation of a thick lens. A precursor beam is used to give uniform pinching to the main beam initially. As the time progresses the pinching becomes non-uniform as shown above. 

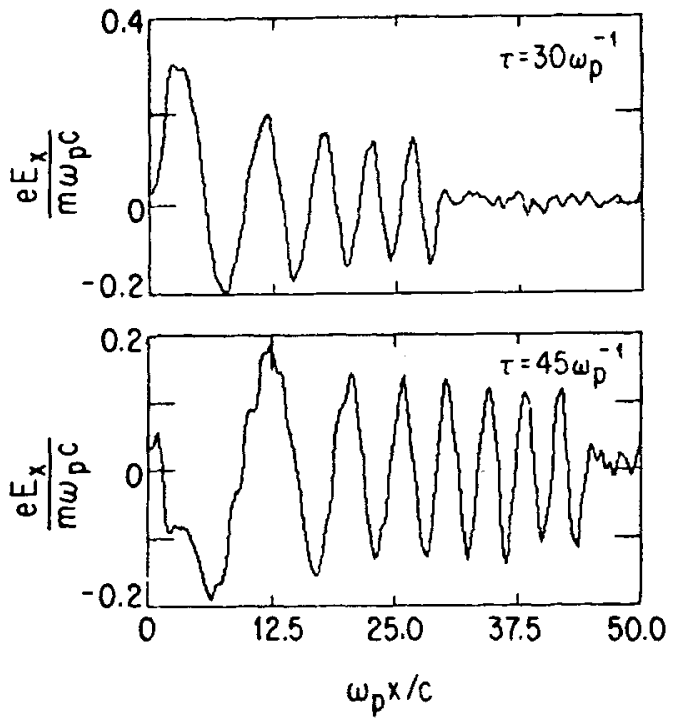

Fig. 18. One possibility for phase-locking the particles in the PWFA is to use a plasma whose density slowly increases. Since the phase velocity of the excited wake is $v_{b}$ irrespective of the plasma density, $\lambda_{b}$ decreases $\omega_{\mathrm{p}}$ increases.

\section{SIMULATIONS PROGRAM}

In this section we present preliminary simulation results on particle injection in the PBWA. Basically four types of simulations were carried out. They are:

- 1-D and 2-D test particle simulations to benchmark behavior for the first low current injection experiments and

- 1-D and 2-D heavy beam loading simulations employing high density shaped bunches to investigate the importance of the various nonlinear effects neglected in the linear analysis presented in section III and refs. (11-13).

The simulations were carried out on $\mathrm{WAVE}^{(15)}$ a 2-D relativistic electromagnetic particlein-cell code. 
Results. We begin with the 1-D simulations. For the low current 1-D simulations the parameters are $T_{e}=1 \mathrm{keV}, \mathrm{L}_{\mathrm{x}}=60 \frac{\mathrm{c}}{\omega_{\mathrm{p}}}, \mathrm{T}_{\text {rise }}=300 \omega_{\mathrm{p}}^{-1}, \omega_{1}=5, \omega_{2}=4, \mathrm{v}_{01} / \mathrm{c}=\mathrm{v}_{02} / \mathrm{c}$ $=.56$ and $\mathrm{m}_{\mathrm{i}} \mathrm{m}_{\mathrm{e}}=\infty$. The parameters were chosen to be identical to our previous 2-D simulations ${ }^{(15)}$ so that the evolution of the plasma wave would be precisely known. The density of the injected beam is $10^{-7}$ of the background and it is injected continuously from $t=90 \omega_{p}^{-1}$ to the end of the simulation. The momentum of the beam is $5 \mathrm{mc}$.

This simulation is summarized in Fig. 19 where the phase space of the background plasma and the injected beam and the electric fields of the laser and the plasma wave are plotted, at $t=150 \omega_{p}^{-1}$. In Fig. 19d the evolution of the plasma wave is shown. The plasma wave is beginning to saturate near the left hand boundary at a value of $\sim 0.5$ $\frac{m c \omega_{p}}{e}$. The wave will eventually reach $0.6 \frac{m c \omega_{p}}{e}$ near $t=160 \omega_{p}^{-1}$. The observed growth behavior is consistent with relativistic detuning when rise time effects are included as we have shown previously ${ }^{(15)}$. In Fig. $19 \mathrm{~b}$ the phase space of the injected beam is presented. The test particles eventually acquire maximum energies consistent with the single particle theory, i.e., $2 \varepsilon \gamma_{\phi}^{2} \mathrm{mc}^{2}$. Additionally, at this time some of the injected electrons are observed to be trapped in the potential buckets. These are the electrons which are a curling back and being reaccelerated after being decelerated. This occurs only after the plasma wave is of a sufficient amplitude.

In the other class of 1-D simulations we inject a high density shaped bunch in the hope of observing the high efficiency predicted in the theory section. The parameters for the lasers and the background plasma are identical to the previous case. The shaped bunch is injected at $t=153.8 \omega_{p}^{-1}$. The density of the bunch falls linearly from .43 of the background to 0 in $1.73 \omega_{\mathrm{p}}^{-1}$. The injection time was chosen near the saturation time of the plasma wave. This will probably not be desirable as we discuss shortly. In Fig. 20 we illustrate that the desired field cancellation is observed initially. The field produced by the injected bunch, the field produced by the lasers and the total field are plotted in Fig. 20a, $b$, and $c$, respectively. Although the electric field produced by the lasers is nonlinear the linear theory given in the previous section appears to be valid because the total field is smaller than without the injected bunch. The field cancellation or equivalently the efficient absorption of plasma wave energy exists only briefly due to phase slippage. The front of the injected bunch only has to phase slip slightly before it sees a significantly different value of the electric field because it initially exists at a point where the derivative of the electric field with respect to the phase is large. Once the front is decelerated the bunch shape is no longer maintained. The phase slippage issue is exacerbated due to the small $\gamma_{\phi}$ $\sim 4.47$ employed. The small value is chosen for computer cost savings. However, for any value of $\gamma_{\phi}$ the point mentioned above needs to be addressed.

To compensate for the phase slippage we injected an identical bunch later in time at $t=155 \omega_{p}^{-1}$. The hope being that the bunch would eventually come into the proper phase of the wave after being only slightly distorted. In the simulation the back of the bunch becomes somewhat monoenergetic and it acquires the predicted final energy of $\gamma \approx$ 13. Another way to avoid phase slippage is to utilize the surfatron concept ${ }^{(16)}$. We have 
BMONJA BEAK LOADINC PBYA AA=.566 IOLAZ=300
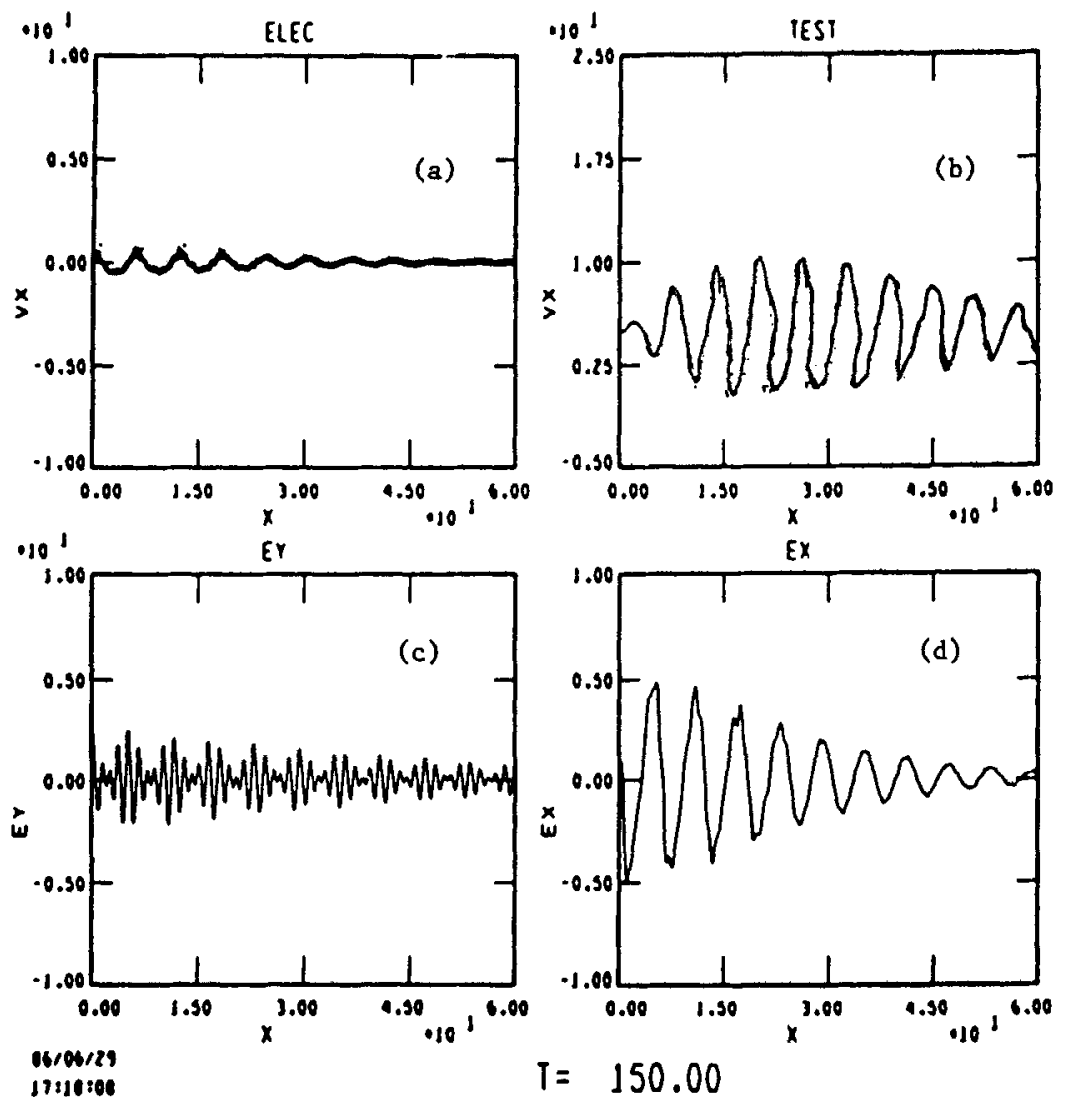

Fig. 19. One dimensional particle simulations of test particle acceleration in the PBWA. (a) Phase space of background electrons.

(b) Phase space of injected electrons with $\gamma=5$. (c) The driving laser beams. (d) The accelerating plasma wave field. 

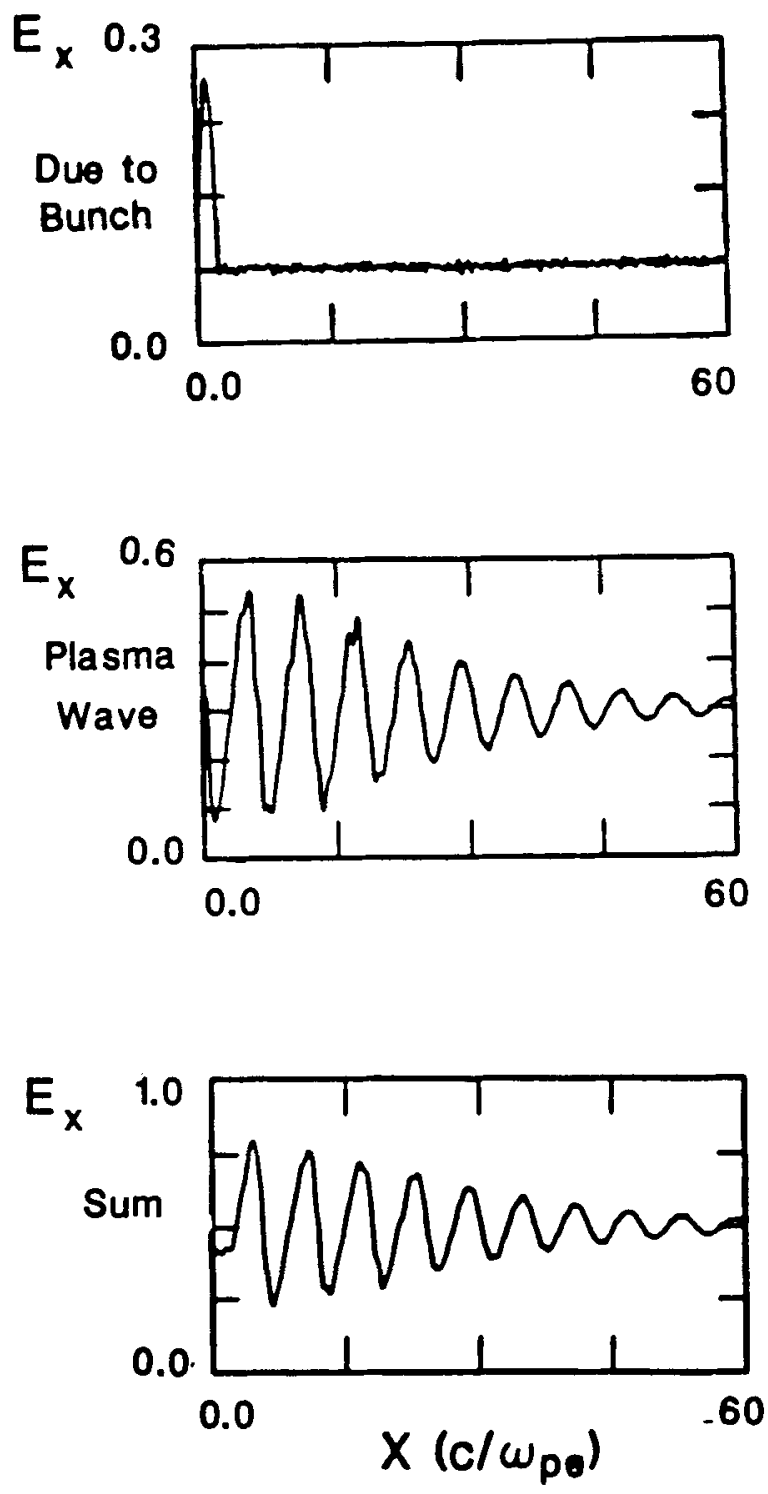

Fig. 20. Beam loading in 1D simulation of the PBWA. (a) Field due to the bunch which is close to the left hand boundary. (b) Field due to the plasma wave. (c) Net field showing depletion due to beam loading at the left hand boundary. 
begun to carry out some simulations to investigate beam loading in the surfatron concept.

Another issue for beam loading in the PBWA is the nonlinear wavelength shift. Just as there is a nonlinear frequency shift there is also a wavelength shift. These occur together, however, such that there is no phase velocity shift to lowest order ${ }^{(17)}$. Since the bunch excites a wave with a fixed wavelength, field cancellation cannot be exact. This problem can be avoided by injecting the bunch before the wave approaches the saturated amplitude.

We next discuss a 2-D simulation in which, as in the first 1-D case, we inject a low density beam of electrons. The parameters were identical to the 1-D case except for the transverse parameters which are $\mathrm{L}_{\mathrm{y}}=60 \mathrm{c} / \omega_{\mathrm{p}}$ and laser beam cross sections of $30 \mathrm{c} / \omega_{\mathrm{p}}$ with a $\cos ^{2} y$ profile for the electric fields. We summarize the results in Fig. 21, where the temporal evolution of the injected electrons $x-y$ phase space is presented. At $t=150 \omega_{p}^{-1}$ the electrons are beginning to get bunched transversely and longitudinally as shown in Fig. 21a. By $t=180 \omega_{\mathrm{p}}^{-1}$ distinct islands are evident as presented in Fig. 21b. The islands form as the result of both 1-D and 2-D effects. In 1-D the electrons are trapped in a particular potential bucket (This is the result of the particular injection energy.). In 2-D focusing and defocusing forces exist, and this has been discussed in the theory section. The trapped electrons only exist in the focusing regions. In addition, the nonlinear saturation occurs at different times for different $y$ positions and this leads to a filamentary structure that is evident in Fig. 21c. The filamentary structure gives rise to more complicated focusing and defocusing forces ${ }^{(17)}$. As a result, the acceleration is not as coherent and this is seen on the left hand sides of Figs. $21 \mathrm{~b}$ and $21 \mathrm{c}$. The above results appear to be in qualitative agreement with theory.

In Fig. 22 we plot the distribution function $f\left(p_{x}\right)$ for the injected electrons. A substantial fraction of the electrons are accelerating. Note that the electrons were injected across the entire box in $y$ while the accelerating field only exist for

$$
20 \frac{c}{\omega_{p}}<y<40 \frac{c}{\omega_{p}} \text {. }
$$

The initial transverse energy spread corresponds to a $\Delta \gamma / \gamma$ of $\sim 10^{-4}$. This spread is much smaller than that expected in the UCLA experiment. Larger transverse energy spreads will decrease the number of accelerated electrons. The reasons for this were discussed in Section III. Simulations with larger transverse energy spreads are being planned.

We conclude with a summary of the heavy beam loading simulation. The bunch had a width of $10 \mathrm{c} / \omega_{\mathrm{p}}$ with a rectangular transverse profile. All other parameters related to the bunch are identical to the 1-D heavy beam loading case and the laser and background plasma parameters are identical to those in the previous 2-D simulation. The simulations illustrate that as in the 1-D heavy beam loading case, some field cancellation is possible. However, phase slippage, the nonlinear wavelength shift and the nonlinear filamentary structure of the plasma wave make agreement with linear theory extremely difficult. We believe that the latter two issues can be avoided by injecting the bunches before the plasma wave becomes nonlinear. When the nonlinearities are absent beam loading in the 

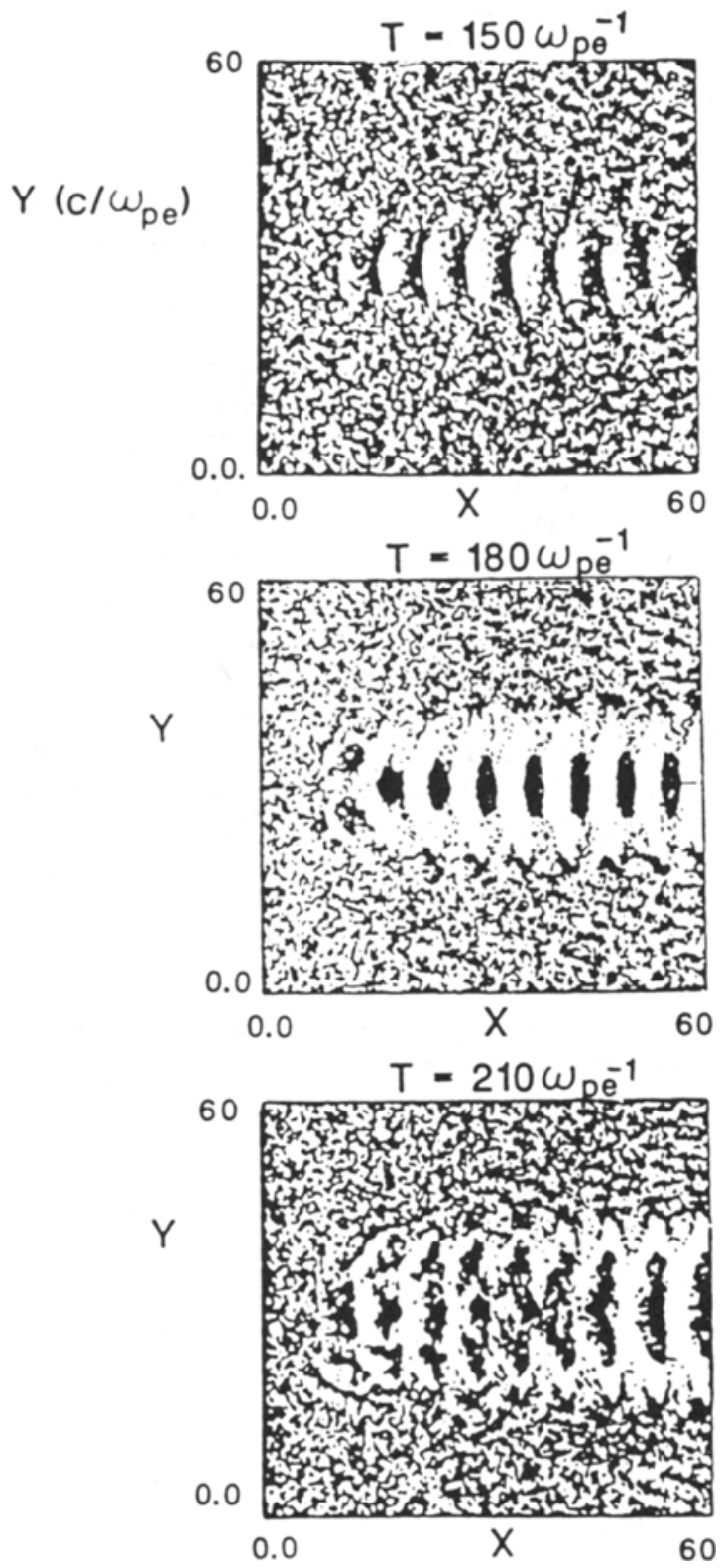

Fig. 21. Two dimensional simulations of test particle acceleration. The test particle positions are shown at various times. 


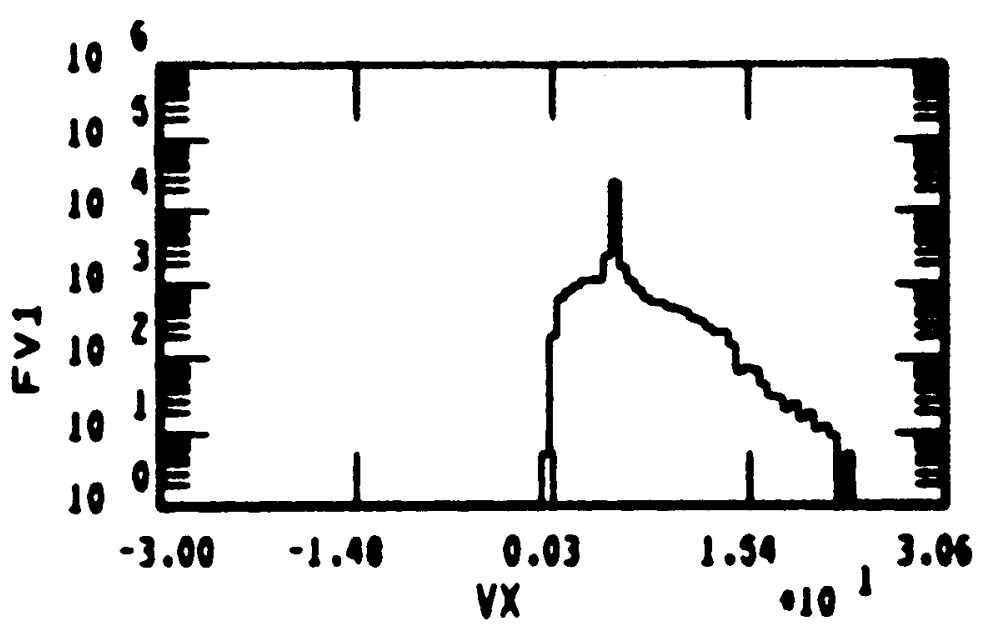

Fig. 22. Distribution function $f\left(p_{x}\right)$ vs $p_{x}$ for the injected electrons.

PBWA and the PWFA become almost identical. However, the issue of phase slippage is unavoidable and needs to be examined in more detail for both schemes.

\section{SUMMARY}

In this paper we have outlined the experimental, theoretical and computational studies in the "Plasma Accelerators" area at UCLA with particular emphasis on the "Plasma Beat Wave Accelerator (PBWA)". The PBWA promises extremely high accelerating gradient through the use of a laser to produce an accelerating space-charge wave in a high density plasma. Such waves have been excited using a $\mathrm{CO}_{2}$ laser and accelerating gradients in excess of $1 \mathrm{GeV} / \mathrm{m}$ have been inferred by light scattering techniques at UCLA. There has been significant parallel progress at INRS (Canada) ${ }^{(10)}$ in detecting the beat excited waves via energy gain of injected particles and at the Rutherford Laboratory $(\mathrm{U} . \mathrm{K})^{(18)}$ in developing the multiphoton ionization technique for producing uniform plasmas.

Theoretical and computational work at UCLA is addressing plasma physics issues as well as the accelerator issues. Numerical and particle code simulations of the upcoming UCLA experiment on the acceleration of test particles by the plasma wave have been carried out. Extensive calculations have been done on the beam loading issue in plasma accelerators. Future work will focus on the laser to plasma wave coupling, so that a zeroorder estimate of the overall efficiency of the PBWA will be possible. 


\section{ACKNOWLEDGMENTS}

This work was supported by DOE contract DE-AS03-ER40120, NSF grant ECS 83-10972, and Los Alamos National Laboratory. Assistance of Ms. Maria Gonzales in preparing this manuscript is acknowledged with pleasure.

\section{REFERENCES}

1. T. Tajima and J. M. Dawson, Phys. Rev. Lett. 43, 267 (1979);

C. Joshi et al., Nature, 311, 525 (1984);

C. Clayton et al., Phys. Rev. Lett. 54, 2343 (1985);

C. Darrow et al., Phys. Rev. Lett. 56, 2629 (1986).

2. P. Chen et al., Phys. Rev. Lett. 54, 693 (1985).

3. J. Rosenzweig et al., see these proceedings.

R. Jones et al., see these proceedings

4. Laser Acceleration of Particles (UCLA), AIP Conf. Proc., No. 130, C. Joshi and T. Katsouleas, eds., (Am. Inst. Phys., New York, 1985).

5. W. B. Mori, UCLA PPG-956 (1986) to appear in the proc. of 1985 CECAM Conf.;

C. J. McKinstrie and D. W. Forslund, Los Alamos Lab Report LA-VR 86-621.

6. C. M. Tang, P. Sprangle, R. Sudan, Appl. Phys. Lett. 45, 375 (1984).

7. J. D. Lawson et al., RAL Report No. RL83057 (1983).

8. R. Ruth, A. Chao, P. Morton and P. Wilson, Particle Accelerators 17, 171 (1985).

9. R. Fedele, U. deAngelis, T. Katsouleas, Phys. Rev. A 33, 4412 (1986).

10. See F. Martin et al., in these proceedings.

11. T. Katsouleas, S. Wilks, P. Chen, J. M. Dawson, J. J. Su, to appear in Particle Accelerators (1986), UCLA PPG-952 (1986).

12. R. Ruth and P. Chen in Proc. 13th SLAC Summer Institute on Particle Physics (1985).

13. S. VanderMeer, CLIC Note No. 3, CERN/PS/85-65(AA) (Geneva, SW, 1985).

14. T. Katsouleas, Phys. Rev. A $\underline{33}, 2056$ (1986).

15. D. W. Forslund et al., Phys. Rev. Lett. 54, 588 (1985).

16. T. Katsouleas and J. M. Dawson, Phys. Rev. Lett. 51, 392 (1983).

17. W. B. Mori, Ph.D. Thesis, UCLA (1987).

18. R. Evans, reported at the Madison meeting. 